\title{
Some Observations on Truth Hierarchies
}

\author{
P. D. Welch, \\ School of Mathematics, \\ University of Bristol, \\ England
}

20.xi.12

\begin{abstract}
We show how in the hierarchies $F_{\alpha}$ of Fieldian truth sets, and Herzberger's $H_{\alpha}$ revision sequence starting from any hypothesis for $F_{0}$ (or $H_{0}$ ) that essentially each $H_{\alpha}$ (or $F_{\alpha}$ ) carries within it a history of the whole prior revision process.

As applications (1) we provide a precise representation for, and a calculation of the length of, possible path independent determinateness hierarchies of Field's construction in [4] with a binary conditional operator. (2) We demonstrate the existence of generalised liar sentences, that can be considered as diagonalising past the determinateness hierarchies definable in Field's recent models. The 'defectiveness' of such diagonal sentences necessarily cannot be classified by any of the determinateness predicates of the model. They are 'ineffable liars'. We may consider them a response to the claim of [4] that 'the conditional can be used to show that the theory is not subject to "revenge problems".'
\end{abstract}

\section{The Scope}

The purpose of this note is to investigate more closely the hierarchies of truth sets produced by the revision sequence process. The first hierarchy, the one produced by Herzberger, [12], [11], was invented to test how various self-referential sentences in a language containing names for elements of a ground model $\mathscr{M}$, and sufficient to define such diagonalising sentences, would behave under repeated applications of the Tarskian definability scheme which produced repeatedly truth sets. Herzberger allowed this process to proceed into the transfinite by using a liminf rule (all of which we specify in more detail below). This revision process has been the subject of various investigations and extensions, notably by Gupta and Belnap in a series of papers, but also in the book [9]. 
More recently Field in e.g. [4], has used such a liminf revision process, to analyse the consequences of adding a binary operator $\longrightarrow$ to a language similar to the above, with $\mathrm{Tr}$ a truth predicate. Field takes at each successive stage not just a new level of definability in the Tarskian sense, but a strong Kleenean fixed point (à la Kripke [15]).

The two sequences of sets we shall dub here $\left\langle H_{\alpha}\right| \alpha \in$ On $\rangle$ (the " $H$-sets") and $\left\langle F_{\alpha}\right| \alpha \in$ On $\rangle$ (the " $F$-sets") (where On denotes the class of ordinals). When defined over the same model, such as $\mathscr{M}=\mathbb{N}$ they are, mathematically at least, surprisingly similar. Indeed we showed in [22] the stability sets consisting of those sentences that are in all the $H$-sets from some point on, and Field's $u l$ timate truth sets are recursively isomorphic - that is there is a pencil and paper algorithm for converting members of one set into the other, and conversely. Of course this is not to say that the members of the final sets are the same or have the same intended meaning. The phenomenon we are seeing here is that the liminf rule is acting as some kind of very powerful infinitary logical rule. One can show that whatever one does (within some considerably wide bounds) at successor steps will be swamped in effect by the limit rule. This is why the two ultimate sets are, up to recursive isomorphism, the same set.

The present paper can be seen as a refinement of this last work where we try and get to grips with the question as to what constitutes each of the $F_{\alpha}$ and $H_{\alpha}$ sets for individual $\alpha$. Such questions seem not to have been really addressed in the literature, but we find we need to analyse what is really happening within these truth-sets and with the liminf rule.

It seems to hard to claim any purely truth-theoretic justification for this rule and on these grounds the present writer finds the revision theories of truth deficient. (To be fair on Herzberger, he made no claims that his methodology was a fully fledged theory of truth; Gupta and Belnap ([9]) on the other hand, claim the rule of revision goes to the very heart of truth, and it is to theories of truth based on such transfinite revision sequences that the above remark is addressed.) Field on the other hand makes no claim that the sets of sentences that are ultimately true are of substantial significance in themselves, or indeed that the construction has some essential features of a theory of truth: it simply provides a model demonstrating the consistency of the kind of principles he would like to have. As he shows, the introduction of a binary $\longrightarrow$ operator renders certain classical principles (such as the law of excluded middle in general) invalid. At the moment we have only a set of principles that are validated by this model's construction (and those of others which he dubs " $G$-models"), but we do not have a theory that is being instantiated by this model. (The same is also true for revision theories.) The situation is rather different from that of Kripke's construction of the Strong Kleene minimal fixed point, which is very clearly tied to a logic, an 
interpretation of connectives, and an axiomatisation.

Martin in [17] in particular, points out that it would be wrong to see Field's construction as playing an analogous role to that of Kripke's for the minimal Strong Kleene fixed point (although Field himself I think is not making this claim, as he does not present this construction as the construction, or as having special status, but only as demonstrating his principles' consistency). Martin, incidentally, also voices doubts about the possibility of any convincing theory of truth that introduces an implication $\longrightarrow$.

Field sees an advantage to his models in that they are able to express the fact that the simple liar $L_{0}$ sentence is somehow defective, being neither in the extension of the Truth or the Falsity predicates, for example in the Kripkean construction, in a way that that Strong Kleene logic cannot. This is done by means of what he calls a 'determinateness operator' and which is a syntactic operation on any sentence $A: D A$ is defined to be $A \wedge \neg(A \longrightarrow \neg A)$. In [4] and [5] the construction allows this to be $A \longrightarrow(\top \longrightarrow A)$ and from this, and the staging process that assigns values to sentences containing $\longrightarrow$, it may be interpreted during the process at a successor stage as " $A$ holds now, and it did at the last stage." This $D$ operation Field iterates, and there is quite a lengthy and difficult discussion in [5] on the lengths of possible iterations of this operator; and how one might iterate it along 'paths', how such paths may be defined, in a bivalent, or a nonbivalent manner etc. This discussion is germane probably to any claim about revenge immunity (which is there for example in the title of [4]) and so it is interesting to see how this unfolds. We believe that, at least in the case of this model's construction, it is possible to give an exact description as to the lengths of such paths that are internally definable within the model. (There is more detail on this outline below.) Furthermore, externally defined paths of longer length will be precisely those for which one is diagonalising out of the model. From this analysis we may define ineffable liars whose defectiveness is beyond capture by any determinateness predicate of the model; thus the attempt to so capture the essence of generalised liars can only work on an initial segment of the definable liar hierarchy.

This all requires a somewhat thorough-going analysis of the mathematics of the model construction process, and thus the $F_{\alpha}$-sets that arise. Hence the main part of this paper is somewhat technical since it perforce must discover these relationships between these sets, and thus the nature of the 'internal' part of the model. This 'internal' is in quotes, since what in fact happens is that a ground model $\mathscr{M}$ such as $\mathbb{N}$ is taken and it is extended to a model $\mathscr{M}^{+}$, in an extended language with ' $T r$ ' and a binary symbol $\longrightarrow$, but which has exactly the same domain of elements. So in what sense can we talk of sets of integers say as being 'internal'? The point is that one can find a formula $A(X)$ with one free variable 
for example, and define $\{n \in \mathscr{M} \mid\|A(X / n)\|=1\}$ where "\|B\|" denotes the ultimate semantic value of a sentence in the construction. In this sense, when using $\operatorname{Tr}(A(X / n))$ as substituted for this $\|A(X / n)\|$, it can be shown that the strong Kleene minimal fixed point has exactly the hyperarithmetic sets of integers as 'internal' to it. One characterisation here is that the internal sets are precisely those that are both themselves and their complements, inductive over that standard model of arithmetic. The models of [4] and [5] also have internal sets, and in particular internal sets defining orderings (and hence 'paths') etc. Once we have constructed such internal paths, then we may safely iterate $D$ along them. Paths defined 'externally' to the model in any way, presumably have no length restrictions, and would correspond to some kind of 'super-determinateness'. We shall further characterise the internal sets in this type of model construction: they form in the case of $\mathscr{M}=\mathbb{N}$ a somewhat large but countable initial segment of the Gödel hierarchy of constructible sets. Then we shall see, taking some terminology from [2], the internal sets in $\mathscr{M}^{+}$are precisely those which together with complements, are 'arithmetically quasi-inductive' sets and occupy the analogous role to the hyperarithmetic sets for the Kripkean construction. Those internal sets that define wellorderings have those orderings' ranks strictly less than some precise bound $\zeta$, defined below, this ordinal taking over the role of the least non-recursive ordinal for the Strong Kleene minimal fixed point.

Our analysis of both the $F$-hierarchy, and the $H$-hierarchy yields complementing results: for any level of the $F$-hierarchy, $F_{\alpha}$ say, has the whole history of the revision process that built it, coded into it. Indeed there is a uniform process, so that given $F_{\alpha}$ the whole sequence $\left\langle F_{\beta} \mid \beta<\alpha\right\rangle$ of prior sets can be retrieved from it ('uniform' meaning that the process is the same for each $\alpha$ ). Moreover this process is arithmetic, so not of great complexity. An entirely analogous result holds for the $H_{\alpha}$ (this is the 'Uniform Definability' result of Lemma 1.3 below). This may perhaps at first sight be surprising. The fact that we can do this is a somewhat delicate set-theoretical matter (which we shall discuss in the rest of the paragraph - although this does not directly affect any of the philosophical consequences). It depends on the fact that the ordinal $\zeta$ concerned, although large proof-theoretically is still in some sense small: it suffices for our purposes that $\zeta \leq \beta_{0}$ where the latter, sometimes called the ordinal of the least model of full second order comprehension, but more commonly for set theorists, is the least ordinal $\beta_{0}$ so that $L_{\beta_{0}} \models \mathrm{ZF}^{-}$- Zermelo-Fraenkel with the power set axiom dropped. Our ordinals are well below that of $L_{\alpha}$ whose reals form the first model of $\Pi_{3}^{1}$-Comprehension (but above that for $\Pi_{2}^{1}$-Comprehension) so we are safely within this region. Nevertheless a set theoretical analysis of the Gödel $L$ hierarchy and how sets are produced is needed: it is precisely because of settheoretical facts that we can establish the uniformity of the arithmetical retrieval 
process from any $F_{\alpha}$.

We have used a part of this 'Uniform Definability' result already in [24]. In order to effect the retrieval of the whole sequence prior to the $\alpha$ 'th stage, it is necessary as a building block, to have first a wellordering of the required length $\alpha$ available. One first establishes that there is such which is also uniformly definable from $F_{\alpha}$ (or $H_{\alpha}$ ). In [24] we were attempting to give a game-theoretic semantics for the Herzberger stability set and the Fieldian ultimate truth set. This was to mirror previous results on the strong Kleenean minimal fixed point by Martin ( $c f$. [16] and [17]) where two players $I$ and $I I$ play a game to determine whether a sentence $A$ was $T$ or $F$ in the fixed point. The possession of a winning strategy by a player indicated that the sentence indeed had a fixed value. If the game were of infinite length then no player had such a strategy and one concludes that neither $A$ nor $\neg A$ is in the fixed point. For the Herzbergerian or Fieldian set, there is indeed such a game but it is necessarily an $\exists \forall \exists$ game, and must in general run for infinitely many moves, even with a winning strategy for a player. This complexity in the game reflects naturally the complexity of the stability and ultimate truth sets involved. However to obtain this characterisation we needed not only that a wellordering of length $\alpha$ was uniformly arithmetic in $H_{\alpha}$ or $F_{\alpha}$, but moreover that it was uniformly recursively enumerable. This observation could then be turned into the result that the $H_{\alpha}$ (and $F_{\alpha}$ ) sets were non-decreasing in $\alpha$. This result was stated but not proved in [24] and we discharge the obligation here.

In general since we now know that there is a close correspondence between the $H_{\alpha}$ and the theories of the $L_{\alpha}$ further results about the Herzberger sequences are perhaps waiting to be mined. For example, one may characterize those levels $H_{\alpha}$ which are models of Cantini's $V F$ : they are precisely those for which $\alpha$ is $\Sigma_{2}$-admissible, or equivalently those $\alpha$ with the reals of $L_{\alpha}$ forming a model of $\Delta_{3}^{1}$-Comprehension (these results may appear elsewhere).

In the next two subsections we outline in more detail these results: in the first the hierarchy theorems we have just discussed, in the second the applications to determinateness hierrachies. In Section 2 we start the construction proper. We first produce these results for the $H$-hierarchy, as there the successor steps are more conventional and perhaps clearly understood. We establish the Uniform Definability and the Non-Decreasing results for this hierarchy. In Section 3 we then see what modifications are needed to claim the same for the $F$-hierarchy. In Section 3.2 we establish our claims concerning path independent hierarchies. Both Sections 2 and 3 depend intrinsically on some analysis of the $L$-hierarchy; these can be treated by the reader uninterested in such technicalities as a black box, and these 'Limit Lemmata' proofs establishing how the theories of various $L_{\lambda}$ (for limit ordinals $\lambda$ ) can be obtained by the liminf process, 
have been hived off to Section 4 . Even if the reader wishes to ignore this section, just some basic knowledge of how the $L$-hierarchy is created will be needed to read Sections 2 and 3. For the results on the Fieldian hierarchy we shall need to assume the reader is familiar with the construction of [4], which is also that of Ch.16. of [5].

\subsection{Truth hierarchies}

Recall that the Herzberger sequence results in a 'loop' that is first entered at stage $\zeta$ and repeats at a later stage $\Sigma$. As established by Burgess [2] the least such pair $(\zeta, \Sigma)$ is the least such pair for which $L_{\zeta} \prec_{\Sigma_{2}} L_{\Sigma}$. We independently established that the universal Infinite Time Turing Machine of [10] also enters a final loop with the same $(\zeta, \Sigma)$ the first such pair (see [23] for an account of this). We first used these two facts to prove the results here on the non-decreasing nature of the Herzberger sequence starting with a null, or any recursive hypothesis or distributions of truth values. We intend here to give direct proofs eliminating the use of machines, and use directly here the, perhaps more familiar, Gödel $L$-hierarchy. We let $H_{\gamma}$ denote the $\gamma^{\prime}$ th truth set over $\mathbb{N}$ of sentences $\sigma$ in the language of arithmetic with an additional $\dot{T}$ symbol to interpret the $H$ sets, $\mathscr{L}_{T}$, using Herzberger's liminf revision rule, and starting out with $H_{0}=\varnothing$. (Any other initial recursive distribution of truth values would have the same effect. Indeed the distribution can be hyperarithmetic or indeed any $H_{0}$ at all, as long as it is an element of $L_{\zeta}$.) Thus we recall:

$$
\begin{aligned}
& H_{\gamma+1}=\left\{\ulcorner\sigma\urcorner \mid\left\langle\mathbb{N},+, \times . \cdots, H_{\gamma}\right\rangle \models \sigma\left[\dot{T} / H_{\gamma}\right]\right\} \\
& H_{\lambda}=\liminf _{\alpha \rightarrow \lambda} H_{\alpha}=\bigcup_{\beta<\lambda} \bigcap_{\beta<\alpha<\lambda} H_{\alpha} \text { if } \operatorname{Lim}(\lambda) .
\end{aligned}
$$

We then have that $H_{\zeta}=H_{\Sigma}=H_{\infty}$ where by the last set we mean the set of sentences stably true in the sequence of length all the ordinals On. $H_{\infty}$ is thus the 'stable truth' set of this process. We demonstrate how, if $\gamma<\Sigma$ then, uniformly in $\gamma$, the whole sequence up to that point, $\left\langle H_{\beta} \mid \beta<\gamma\right\rangle$, is arithmetically obtained from $H_{\gamma}$ (Lemma 1.3 below). We use a part of this result to show:

Theorem 1.1 ( $H$-Non-Decreasing)

$$
\text { If } \beta<\gamma<\Sigma \text {, then in the Herzberger revision sequence } H_{\gamma} \nsubseteq H_{\beta} \text {. }
$$

The same methods can be used to show that for Field's construction in [4] which we showed in [22] essentially constructed a recursively isomorphic copy of the stability set $H_{\zeta}$ of the Herzberger sequence, that we can say the same for his sets. 
Field does the following (particularising to the case of building truth sets over the structure of the natural numbers $M=\langle\mathbb{N},+, \times 0, T\rangle$ ).

Each new model $M_{\alpha}$ only has the extension of the truth predicate, and the extension of the operator $\rightarrow$ changed, and $M_{\alpha, \sigma}$ assigns semantic values from $\left\{0, \frac{1}{2}, 1\right\}$ to sentences. $M_{\alpha, \sigma+1}$ is then the strong Kleenean jump of $M_{\alpha, \sigma}$ according to the usual truth tables. A Kleenean fixed point stage has been reached when $M_{\alpha, \sigma}=M_{\alpha, \sigma+1}$, denoted $M_{\alpha, \Omega}$, which is essentially the usual strong Kleene fixed point computed over the starting model $M_{\alpha, 0}$ with a fixed assignment of values to the conditional. At such starting stages $M_{\alpha, 0}$ and all subsequent stages $M_{\alpha, \sigma}$ up to the next fixed point, conditionals are assigned values as follows according to a revision-theoretic liminf rule:

$$
\begin{array}{rrr}
|A \rightarrow B|_{\alpha, \sigma} & =1 & \text { if } \exists \beta<\alpha \forall \gamma \in[\beta, \alpha)\left(|A|_{\gamma, \Omega} \leq|B|_{\gamma, \Omega}\right) \\
& =0 & \text { if } \exists \beta<\alpha \forall \gamma \in[\beta, \alpha)\left(|A|_{\gamma, \Omega}>|B|_{\gamma, \Omega}\right) \\
& =\frac{1}{2} & \text { otherwise. }
\end{array}
$$

We shall freely use the notion of ' $|A|_{\beta}$ ' (as Field does) for $|A|_{\beta, \Omega}$. For our purposes here, we may define for $\beta<\Sigma$ :

$$
F_{\beta}=d_{d f}\left\{\langle\ulcorner A \longrightarrow B\urcorner, 1\rangle:|A \longrightarrow B|_{\beta}=1\right\} \cup\left\{\langle\ulcorner A \longrightarrow B\urcorner, 0\rangle:|A \longrightarrow B|_{\beta}=0\right\} .
$$

Because of the liminf rule, we thus have for limit $\lambda$ that $F_{\lambda}$ includes codes for those sentences $A$ that either stably have semantic value 1 below $\lambda$, or stably have value 0 . (To see this just look at any $A$, and see if $\langle\ulcorner\top \longrightarrow A\urcorner, 1\rangle$ is in $F_{\lambda}$ etc. ) Similarly from $F_{\alpha+1}$ one may read off the sentences $A$ that had value 1 (or 0 ) at the previous stage: $|A|_{\alpha, \Omega}=1$ (0 respectively). Indeed from $F_{\alpha}$ one may read off all the values $|A \longrightarrow B|_{\alpha}$, and thus all the semantic starting values necessary for calculating the next Strong Kleene fixed point over those values, in this construction. Those fixed point values are then written into $F_{\alpha+1}$ as defined above.

Because of the same limit rule, the stability sets $F_{\zeta}$ and $H_{\zeta}$ are very much the same mathematically speaking, and the sequences can be analysed in somewhat similar fashions. Field's first 'acceptable point' $\Delta_{0}$ of his sequence was shown in [22] to coincide with $\zeta$, and the second with $\Sigma$. (It is a feature of these kinds of inductive sequence, that the limit stages are determined by the liminf rule, which is in effect some form of infinitary rule; and this wipes out differences in what one does at successor stages; one could even have much stronger (or weaker) successor stage operations than Field considers, but if we stick with the liminf rule at limits one again ends up with the same pair of 'stability' ordinals $(\zeta, \Sigma)$ reappearing. ${ }^{1}$ We then have analogously to the above:

\footnotetext{
${ }^{1}$ In [7] he considers changing the conditional $\longrightarrow$. We have not checked but strongly conjecture
} 
Theorem 1.2 ( $F$-Non-Decreasing)

$$
\text { If } \beta<\gamma<\Sigma \text {, then in the Fieldian sequence } F_{\gamma} \nsubseteq F_{\beta} \text {. }
$$

We don't know if there is a simpler direct method of establishing either of these Non-Decreasing Lemmata. Essentially the original single motivating idea can be expressed as follows. Firstly, since the $H$ sets encompass iterated definability, then they should (and do) encode the levels of the $L$ - hierarchy which is also defined by iterated definability along the ordinals. Secondly, we are sufficiently low down in the $L$-hierarchy, that the levels are all the ranges of maps with partial domain $\omega$ which themselves are simply defined over those levels. In particular there are simply defined wellorderings of order type the height of the structure, definable over the structure itself. (Simple here has a technical meaning.) If $\beta<\gamma$ are sufficiently closed ordinals, then one should be able to effectively decode a wellordering $w_{\gamma}$ of type $\gamma$ from $H_{\gamma}$. Lastly, if this decoding is effective enough, and the wellordering $w_{\beta}$ of type $\beta$ is decodeable from $H_{\beta}$ in the same way, then this will prevent $H_{\gamma}$ being a subset of $H_{\beta}$. That is the idea.

Pushing these ideas further we shall in fact have something more:

Lemma 1.3 'Uniform Definability' (i) There is a single uniform method of arithmetically defining the whole sequence $\left\langle H_{\gamma} \mid \gamma<\beta\right\rangle$ from $H_{\beta}$ for any $\beta<\Sigma$. Again this method is uniform in the sense that it is independent of $\beta$.

(ii) The same as (i) with the Fieldian sets $F_{\gamma}$ replacing $H_{\gamma}$.

In the case of a successor $\beta=\gamma+1<\Sigma$ we may moreover assert that there is a single recursive function (thus independent of $\beta$ ) $F: \mathbb{N}^{2} \longrightarrow \mathbb{N}$, so that if we set

$$
\mathscr{H}=\left\{\langle\ulcorner A\urcorner, u\rangle \in \mathbb{N}^{2} \mid F(\langle\ulcorner A\urcorner, u\rangle) \in H_{\beta}\right\}
$$

then with $w_{\beta}$ the well ordering of type $\beta$ of the type sketched above, and $u \in$ Field $\left(w_{\beta}\right)$, then, if $u$ has rank $\gamma$ in $w_{\beta}$ then $\mathscr{H}_{u}=_{\mathrm{df}}\{\ulcorner A\urcorner \mid\langle\ulcorner A\urcorner, u\rangle \in \mathscr{C}\}$ is nothing other than $H_{\gamma}$ itself. Thus for such $\beta$ we have a way not only of defining simply a wellorder of type $\beta$ from $H_{\beta}$, but we may recursively recover the whole prior sequence $\left\langle H_{\gamma} \mid \gamma<\beta\right\rangle$ from knowledge of $H_{\beta}$. Again the method is independent of $\beta$. Hence we may think of $H_{\beta}$ as always encoding the whole revision sequence up to $\beta$. From a set-theoretical perspective, this is just as it should be. For limit $\beta<\Sigma$ the process is more complicated: it is still arithmetical rather than recursive, but still can be done uniformly. Again the same is true for the

that for this notion the very same ordinals $\zeta, \Sigma$ are relevant: again this is symptomatic of this kind of strong infinitary rule. 
$F$-sequence. This Lemma represents the content of the second paragraph of our abstract.

It is from the Uniform Definability that we get a special kind of reflection in our sequences: we shall see that any talk about stabilization (or otherwise) of a formula $B$ in a hierarchy, can itself be expressed, or reflected, by formulae about, inter alia, a code of $B$, that themselves stabilize (or otherwise). This will be put to use in particular in the next subsection and Section 3.2.

\subsection{Determinateness Hierarchies}

Field has defined a notion of determinateness that seeks to express the idea that whereas some sentences (such as a simple liar $L_{0}$ ) in, for example, a Strong Kleenean fixed point are neither true nor false, that language lacks the expressiveness to somehow qualify that liar sentence as having that intermediate status. In his model of [4] he considers for each sentence $A$ a corresponding sentence asserting the determinate truth of $A$. There it is $A \wedge(T \longrightarrow A)$. This he abbreviates as $D A$. In his construction the ultimate value of the simple liar $\left\|L_{0}\right\|$ is $\frac{1}{2}$, whereas $\left\|D L_{0}\right\|$ is easily seen to be 0 . In turn $\| \neg D \operatorname{Tr}\left(\left\ulcorner L_{0}\right\urcorner \|\right.$ has value 1 , and thus we may say that although we cannot assert that the liar $L_{0}$ is not true we can say that it is not determinately true. We thus have the means to express to some extent the 'defectiveness' of the liar in not having a $0 / 1$ semantic value. By the usual diagonal argument there is however a sentence $L_{1}$ expressing $\neg D \operatorname{Tr}\left(\left\ulcorner L_{1}\right\urcorner\right)$. Again $\left\|L_{1}\right\|$ is $\frac{1}{2}$ but so is $\left\|D L_{1}\right\|$. Basically this is because, whereas the simple liar $L_{0}$ alternates in value from 0 to 1 or back again at every stage, $D A$ - which asserts " $A$ now and $A$ was true at the previous stage" (to paraphrase: we took $T \longrightarrow A$ to express the latter conjunct) when $D$ is applied to $L_{0}$ this must be static at zero. Change the periodicity of the alternation, say from every stage to every two stages - as is the case with $L_{1}$ - then $D L_{1}$ will itself switch from 0 to 1 and back again, switching from 0 to have value 1 every fourth stage. However instead $D D L_{1}$ can be seen to have value 0 everywhere. Defining $L_{2}$ to be equivalent to $\neg D^{2} \operatorname{Tr}\left(\left\ulcorner L_{2}\right\urcorner\right)$ a similar analysis holds, where now $L_{2}$ has a periodicity 3 . Field then defines iterations $D^{n} A$ in the obvious way and a transfinite iteration $D^{\omega} A$ is taken as (the formal version of) “ $\forall n \operatorname{Tr}\left(\left\ulcorner D^{n} A\right\urcorner\right)$ )". We may then define $D^{\omega+1} A$ as $D D^{\omega} A$ and so forth, For each $D^{\alpha}$ so defined there is a generalised liar $L_{\alpha}$ with, amongst others, the properties that $\left\|L_{\alpha}\right\|=\frac{1}{2}=\left\|D^{\alpha} L_{\alpha}\right\|$ but $\left\|D^{\alpha+1} L_{\alpha}\right\|=0$.

Field asks then for how long this process may continue. In [4] he mentions that this can be done at least up to some recursive ordinal $\lambda_{0}$. In [6] it is remarked that this is too restrictive and that it can be done for all recursive ordinals. In the latter paper and the book [5] there are lengthy discussions as to how to define first 'path dependent hierarchies' of the $D$ operator, and even 'path independent 
hierarchies'. In essence one wants a path of iterations of $D$, and for finite ordinals, or recursive ordinals, there are orderings readily to hand along which to effect this. (For recursive orderings there are the Kleene $\mathscr{O}$ notations to 'name' ordinals below $\omega_{1}^{\mathrm{ck}}$ - the first non-recursive ordinal, to effect this - $c f$. [21].) Field would like the iterations of the ' $D$-operator' to lead to concepts and notions of determinateness of increasing strength, but if these notions depended on the path (read: ordering or ordinal notation system) used, this is rather undesirable. What we want are 'path independent hierarchies' which lead to notions so independent. There is some difficult discussion on this, but it seems that, at least for the principal model under discussion or maybe its counterpart when the ground model is not arithmetic, but some model of set theory of the form $V_{\mathcal{K}}$ - the collection of sets of rank less than some ordinal $\kappa$, the upshot is that such hierarchies are of some unspecified, or 'fuzzily defined' length which 'fall short of the first acceptable point' ([6]).

It is part of our task (which we sketched in [25]) to bring some clarity to this discussion, at least for models of the kind described in [4] and [5]. Here this 'principal model' construction allows one to internally define paths in the model $\mathscr{M}^{+}$up to $\Delta_{0}(\mathscr{M})$ the first 'acceptable ordinal' over the model. We thus end up with two tasks, to establish a) how to explicitly get such paths - in essence bivalently defined prewellorderings and $b$ ) an explicit and exact upper bound on the lengths of such.

One might ask whether that has exhausted the possible 'path independent hierarchies' that Field envisages, but we see no sensible mechanism for this beyond what we have proposed. Could we then claim that we have listed all possible notions of strengthened determinateness? Indeed one of our results below (Lemma 1.7) explicitly says that there are no paths at all of the kind we describe that are longer than ours. Hence there are no such internally defined notions of determinateness beyond, or stronger than, what we have produced here. It would seem then that an externally defined path of length longer than $\Delta_{0}$ is just what one does not want: from that one can define all the internal paths and could then easily diagonalise out of the sets defined from the model.

Proposition 1.4 There are sentences $C \in \mathscr{L}^{+}$so that for any determinateness predicate $D^{B}$ with $B \in$ Field $(\preceq)\left\|D^{B}\left(L_{C}\right)\right\|=\frac{1}{2}$. Thus the defectiveness of $L_{C}$ is not measured by any such determinateness predicate definable within the $\mathscr{L}^{+}$language.

This is proven in the final subsection of Section 3. These are our examples of diagonalised sentences whose defectiveness is not encompassed by any $D^{B}$ for $B$ genuinely in Field( $(\leq)$ : they are the ineffable liars. 
In the Kripkean construction over the standard model of arithmetic one can define for any sentence $A, \rho_{0}(A)$ to be the least ordinal $\alpha$ (if it exists) so that $A \in \Phi_{\alpha+1}$, where $\left\langle\Phi_{\beta} \mid \beta<\omega_{1}^{c k}\right\rangle$ enumerates the stages building up the Strong Kleenean fixed point. We may define a formula $P_{0}\left(v_{0}, v_{1}\right)$ in $\mathscr{L}_{\dot{T}}$ so that if $\rho_{0}(B) \downarrow$ and if $A$ is any formula, the $P_{0}(\ulcorner A\urcorner,\ulcorner B\urcorner)$ has a definite semantic value of 0 or 1 and then $\rho_{0}(A)<\rho_{0}(B)$. We do something similar here using the idea of a sentence becoming stably true (or false) rather than becoming simply true or false in terms of the extension and anti-extension of the Kripkean construction. This is the idea behind the function $\rho$ and formula $P_{<}$of the next paragraph.

For a sentence $A$ we may define $\rho(A)$ to be the least ordinal $\rho$ (if it exists) in a revision sequence so that the semantic value of $A$ is constant from stage $\rho$ onwards. We may define in the language $\mathscr{L}^{+}$a prewellordering $<$of sentences of stabilizing truth value: we set $P_{<}(\ulcorner A\urcorner,\ulcorner B\urcorner)$ if and only if $\rho(A)<\rho(B)$, where $\ulcorner A\urcorner$ is an integer Gödel code for $A$. (It has to be shown that we can do this and that $P_{<}$is given by an $\mathscr{L}^{+}$formula.) We could do this just for sentences stabilizing just on 1 , or on 'designated truth values', but we do this here for 0,1 only. The ordering $\leq$ derived from $\prec$ is a prewellordering since naturally many sentences $A$ may stabilize at the same ordinal. Letting $\|A\|$ be the ultimate semantic value of the sentence $A$, in the model $\mathscr{M}^{+}$, we then show:

Lemma 1.5 There are formulae $P_{\leq}\left(v_{0}, v_{1}\right), P_{<}\left(v_{0}, v_{1}\right)$ in $\mathscr{L}^{+}$so that for any sentences $A, B \in \mathscr{L}^{+}$, we have

$$
\begin{aligned}
\left\|P_{<}(\ulcorner A\urcorner,\ulcorner B\urcorner)\right\| & =1 \text { iff } \rho(A) \downarrow, \rho(B) \downarrow \text { and } \rho(A)<\rho(B) ; \\
& =0 \text { iff } \rho(A) \downarrow, \rho(B) \downarrow \text { and } \rho(A) \geq \rho(B) ; \\
& =\frac{1}{2} \text { otherwise. }
\end{aligned}
$$

(And similarly mutatis mutandis for the formula $P_{\leq}$.)

The construction of these formulae $P_{<}$and $P_{\leq}$will build on the work of the above. We abbreviate $A<B$ for $\left\|P_{<}(\ulcorner A\urcorner,\ulcorner B\urcorner)\right\|=1$ etc. Then, if $\|A\|=1$ (or 0 ) say, then $\{B: B<A\}=\left\{B:\left\|P_{<}(\ulcorner A\urcorner,\ulcorner B\urcorner)\right\|=1\right\}$ is a prewellordering of order type some ordinal $\xi<\Delta_{0}$. It is less than $\Delta_{0}$ since, recall, that any sentence that stabilizes must do so by $\Delta_{0}$ by the latter's definition.) We let Field $(\prec)$ denote the set of sentences stabilizing on 0 or 1 . The next lemma shows how long these prewellorderings can be:

Lemma 1.6 For any $\xi<\Delta_{0}$ there is a sentence $A=A_{\xi}$ in Field $(<)$ with the order type of $\{B \mid B<A\}$ equalling $\xi$.

That this is as far as one can go is shown by: 
Lemma 1.7 Let $Q\left(v_{0}, v_{1}\right)$ be a formula of $\mathscr{L}^{+}$. Define $n \prec_{Q} m$ if $\|Q(n, m)\|=1$. Suppose $\prec_{Q}$ is a prewellordering, and further that for any $m \in \operatorname{Field}\left(<_{Q}\right)$, it is a bivalent matter for any $n \in \mathbb{N}$ whether $Q(n, m)$. Then $\operatorname{ot}\left(<_{Q}\right) \leq \Delta_{0}$.

The assumptions are thus that $Q$ defines a prewellordering, $\prec_{Q}$, so that, to rephrase, for any $m \in \operatorname{Field}(<)$, for any $n \in \mathbb{N}\|Q(n, m)\| \neq \frac{1}{2}$. The bound of $\Delta_{0}$ is attained by the ordering $P_{<}$above. This then delimits the kind of determinateness hierarchies of the kind we have been considering to have lengths strictly less than $\Delta_{0}$.

We now have the wherewithal to define internal hierarchies of iterated determinateness along initial segments of $<$ given by the sets $\{B: B<A\}$. We may define for any sentence $C$ :

$$
D^{C}(A) \equiv \forall B\left(P_{<}(B, C) \rightarrow\left(\forall y\left(y=\left\ulcorner D^{B}(A)\right\urcorner \rightarrow T(y)\right)\right)\right) .
$$

For $C \in \operatorname{Field}(\leq)$ this defines a 'genuine' determinateness hierarchy of length $\rho(C)$. However it is not a bivalent matter as to whether a general $C$ is or is not in Field $(\preceq)$. (In other words Field $(\preceq)$ is not a crisp subclass of $\mathbb{N}$.) However if $C \in \operatorname{Field}(\preceq)$ then it can be shown that it is a bivalent matter whether a general $B$ is <-below $C$ or not (Lemma 3.8 below).

Because of the presence of sentences $C$ for which we cannot bivalently assign a $0 / 1$ semantic value to " $C \in$ Field $(\preceq)$ " the expression " $\left\langle D^{B}\left(\nu_{0}\right) \mid B<C\right\rangle$ forms a determinateness hierarchy" is not in the classical part of the language $\mathscr{L}^{+}$to which the Law of Excluded Middle holds. I believe that this gives a precise formulation to Field's idea that ' $\mathrm{O}$ is an iteration of $D$ is 'fuzzy' ' in this context ([6], Sect.17). Lemmas 1.6 and 1.7 give the extent of such hierarchies.

The 'ordinals internally $\mathscr{L}^{+}$-definable' are thus for us the ordinals $\xi<\Delta_{0}$, which we define through our use of stabilizing sentences and the ordering $\leq$. Although the latter has order type precisely $\Delta_{0}$ (by Lemma 2) there is no sentence $\delta$ stabilizing precisely at stage $\Delta_{0}$. Thus the internally defined determinateness hierarchy breaks down, not fuzzily, but precisely, at $\Delta_{0}$. There is no internally definable maximal hierarchy. Externally we see exactly what is going on, and could of course, define a hierarchy of length $\Delta_{0}$ using the full field of the ordering $<$.

If one takes the formula $P_{<}$, then for any ordinal $\delta$ with $\Delta_{0}<\delta<\Sigma$ (where $\Sigma$ is the next acceptable point above $\Delta_{0}$ ) there will be an ordinal $\xi$ with $\delta<\xi<\Sigma$, and there will be $C$ so that $\left\{B:|B<C|_{\xi}=1\right\}$ is a prewellordering under $\prec$ of order type $\delta>\Delta_{0}$ and further, defining $D^{C}(A)$ as above:

$$
\mid D^{C}(A) \text { forms a determinateness hierarchy }\left.\right|_{\xi}=1 .
$$


However this is only an evaluation at a non-acceptable point $\xi$, and the semantic value of such when evaluated at $\Delta_{0}$ or $\Sigma$ is quite different, as it must be by Lemma 1.7.

There is some discussion in [6] (see, e.g. Sect. 12) about the plausibility, or otherwise, of defining $D^{\alpha}$ operators that somehow with the index $\alpha$ considered as a variable, allow one to define an operator that quantifiers over all possible $\alpha$. We have defined $D^{C}$ for any sentence $C$. One might be tempted to quantify over all $C$ and thereby introduce some further hyper-operator. The discussion of the last paragraph shows this to be fruitless, since the $D^{C}$ for unstabilized $C$ are not really determinateness hierarchies. However we have no need to do anything like this: the ineffable liar sentences $D^{C}\left(L_{C}\right)$ already escape being measured in their defectiveness by any determinateness operator expressible within the model.

Thus, viewing the construction of the model dynamically, there are longer paths, hierarchies, prewellorders etc, but they are evanescent: they appear for a while in the revision process, but then disappear: $\Delta_{0}$ is the sum total of all the hereditarily definable ordinals. It is the least 'fuzzy' ordinal in that it is the least ordinal which is not the length of a 'stabilized' or 'bivalently defined' or 'internal' wellordering.

Acknowledgements: I should like to thank Leon Horsten, Graham Leigh, and Toby Meadows for their comments on an earlier draft, but most particularly the referee for his cogent suggestions for a substantial improvement to the presentation of the technical arguments.

\section{The construction}

We shall be able to conclude that for all limit ordinals $\beta$, that there is always a wellordering of $\mathbb{N}, w_{\beta}$, of order type $\beta$ which is recursively enumerable in $H_{\beta}$, uniformly in $\beta$. Here 'uniformly' means that the definition does not depend on $\beta$ but is the same for all limit $\beta$ less than $\Sigma$ (the fact that there is such a definition at all depends crucially on the defining property of $\Sigma$ ). In slightly finer detail it will be asserted that there is a recursive (1-1) function $G: \mathbb{N} \times \mathbb{N} \longrightarrow \mathbb{N}$, so that if $w_{\beta}=\left\{\langle u, v\rangle \in \mathbb{N} \mid \exists i \in \mathbb{N} G(i, \prec u, v>) \in H_{\beta}\right\}$ then $w_{\beta}$ is an ordering of type $\beta$, for $\operatorname{Lim}(\beta)$. Now, towards a proof of Theorem 1.1, if $\beta<\gamma<\Sigma$ are both limits, and we supposed that $H_{\gamma} \subseteq H_{\beta}$, then $G^{-1 \text { " }} H_{\gamma} \subseteq G^{-1}$ " $H_{\beta}$. However this would be absurd as then we should have $w_{\gamma} \subseteq w_{\beta}$ and thus $w_{\beta}$ has a suborder of type $\gamma$ ! This is the contradiction. This proof then depends on the construction of $G$ which, perhaps surprisingly, turns out to be not a trivial matter. We also have 
the minor irritant of having to deal with those ordinals $\beta, \gamma$ etc. not limits. This we shall get by noting that not only is $H_{\lambda} \leq_{T} H_{\beta}$ (for any $\beta$ with $\lambda \leq \beta<\lambda+\omega$ ) but there is in fact a uniform way independent of $\beta$ and $\lambda$, of (1-1) recursively reducing any such $H_{\lambda}$ to any such $H_{\beta}$.

In fact it is possible to regard this paper as chiefly about the construction of two recursive functions, a $G=G_{H}$ just described, and another $G_{F}$ for the Fieldian hierarchy. The way this has been achieved is to demonstrate that the $L$-hierarchy $\left\langle L_{\alpha} \mid \alpha<\lambda\right\rangle$ is uniformly arithmetical in $H_{\lambda}$. Then from known facts about the $L$-hierarchy, we deduce the existence of the required wellorderings $w_{\lambda}$ etc.

We have taken $<-,->: \mathbb{N} \times \mathbb{N} \longrightarrow \mathbb{N}$ to be some fixed recursive bijection. We shall further use standard terminology from recursion theory. We shall use

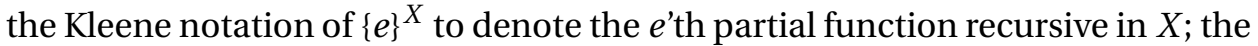
domain of this function is denoted $W_{e}^{X}$. We shall as usual write $A \leq_{T} B$ to mean that $A$ is Turing reducible to $B$, which in turn means that the characteristic function of $A$ is recursive in $B . A \leq_{1} B$ will indicate that $A$ is (1-1) reducible to $B$ : there is a total recursive (1-1) function $f: \mathbb{N} \rightarrow \mathbb{N}$ so that $A=f^{-1}$ " $B$. We shall quote without further specifying here standard theorems, such as the snm-theorem and the (Second) Recursion Theorem (for these and all other facts of this paragraph see either [20] or [19]). We note that for any $X, K^{X}={ }_{d f}\left\{e \mid e \in W_{e}^{X}\right\}$, is a complete $\Sigma_{1}^{X}$ set (being $\Sigma_{1}$-definable over $\langle\mathbb{N}, X\rangle$, and all sets $Y$ recursively enumerable (r.e.) in $X$ are (1-1) reducible to it). We set $X^{(0)}=X$ and let $X^{(1)}={ }_{d f} X^{\prime}$, the Turing jump of $X$, to be this set $K^{X}$, and let $X^{(n+1)}$ be $\left(X^{(n)}\right)^{\prime}$. Let $X^{(\omega)}=d f$ $\left\{\langle n, k\rangle \mid k \in X^{(n)}\right\}$. Then $X^{(\omega)}$ is the complete arithmetic set over $X$. Recall also that if $X \leq_{T} Y$ then $X^{(\omega)} \leq_{1} Y^{(\omega)}$. In our context we have that for $n \geq 1$ that $\left(H_{\alpha}\right)^{(n)}$ is (1-1) reducible to the complete $\Sigma_{n}$ theory of $\left\langle\mathbb{N}, H_{\alpha}\right\rangle$. Further, $\left(H_{\alpha}\right)^{(\omega)}$ is (1-1) reducible to $H_{\alpha+1}$ (in particular for any $e W_{e}^{H_{\alpha}} \leq_{1} H_{\alpha+1}$ ). We let $G_{1}$ be a recursive function witnessing this last reduction.

Lemma 2.1 (i) There is an effective procedure for testing $H_{\beta}$ to determine if $\beta$ is a multiple of $\omega$. (ii) For $\omega>n \geq 0$ there is a sentence $\tau_{n}$ so that $\operatorname{Lim}(\lambda) \rightarrow(\beta=$ $\left.\lambda+n+1 \leftrightarrow \tau_{n} \in H_{\beta}\right)$.

Proof: Firstly we note that we can always tell from $H_{\beta}$ whether $\operatorname{Lim}(\beta)$ or not: we look and see if both $L_{0}$ and $\neg L_{0}$ are absent from $H_{\beta}$ where $L_{0} \leftrightarrow \neg T\left(\left\ulcorner L_{0}\right\urcorner\right)$ is a simple Liar sentence. By the Herzberger rules, this happens precisely at limit $\beta$. Let $\tau_{0}$ be the sentence $\neg T\left(L_{0}\right) \wedge \neg T\left(\neg L_{0}\right)$. Then $\tau_{0}$ is true in $\left\langle\mathbb{N}, H_{\mu}\right\rangle$ (and hence is in $\left.H_{\mu+1}\right)$ iff $\operatorname{Lim}(\mu)$. Now set for $\left.n \geq 1, \tau_{n} \equiv T^{n}\left(\tau_{0}\right)\right)$. Then for $n \geq 1$, $\left\langle\mathbb{N}, H_{\mu}\right\rangle \vDash \tau_{n}$ iff $\mu=\lambda+n$ where $\lambda$ is the largest limit less than or equal to $\mu$.

Q.E.D. 
Lemma 2.2 (i) There is a (1-1) total recursive function $f_{0}$ so that for any limit $\lambda$ and any $n<\omega$, then $H_{\lambda}=f_{0}^{-1}$ " $H_{\lambda+n+1}$.

(ii) Moreover the sequence $\left\langle H_{\lambda+k} \mid 0 \leq k<n\right\rangle$ is uniformly recursive in $H_{\lambda+n}$ for any such $\lambda$ and $n \in \mathbb{N}$.

Proof: There is an effective list of indices $\mathscr{E}=\left\langle e_{k} \mid k<\omega\right\rangle$ for recursive functions $F_{k}$, with the property that for $k>0, e_{k}$ is an index of the function $F_{k}$ so that $F_{k}(s)$ is the gödel code of the result of adding $k$ applications of $T$ to the sentence with gödel code $s$. (Here $F_{0}=\left\{e_{0}\right\}$ is taken as the identity function.) Let $f$ be the following function, which is recursive in $X \subseteq \mathbb{N}$ :

$$
\begin{aligned}
& f(s)=1 \text { if } s \in X, L_{0} \notin X \text { and } \neg L_{0} \notin X \text { or } \\
& \text { if } F_{k+1}(s) \in X \text { where } k \text { is least so that } \tau_{k} \in X ; \\
&=\uparrow \quad \text { otherwise. }
\end{aligned}
$$

Then for some index $e$ of the function $f$, if $X=H_{\lambda+k}$ for any $k<\omega, H_{\lambda}=$ $W_{e}^{X}$. But in general $W_{e}^{X}$ is (1-1) reducible to $K^{X}$. That is for some total recursive $G, W_{e}^{X}=G^{-1 \text { " }} K^{X}$. We combine this with the fact that for any $\beta<\Sigma$, there is a total recursive function $h$ witnessing $K^{H_{\beta}} \leq_{1} H_{\beta+1}$ (this is because $K^{H_{\beta}}$ is $\Sigma_{1}$ definable over $\left\langle\mathbb{N}, \cdots, H_{\beta}\right\rangle$ ). We take $f_{0}=h \circ G$. This finishes (i). (ii) is similar. We shall show that there is a (1-1) recursive partial function $h^{X}: \mathbb{N} \times \mathbb{N} \rightarrow \mathbb{N}$, partial recursive in any set $X$, so that for any limit $\lambda \leq \Sigma$, and for any $n \in \mathbb{N}$, if $X=H_{\lambda+n}$, then $h^{X}$ is total, and $H_{\lambda+k}=\left\{s \mid h^{X}(k, s)=1\right\}$ for $k<n$.

Define

$h(k, s)=1 \leftrightarrow$ for the least $n$ such that $\tau_{n-1} \in X\left\{e_{n-k}\right\}(s) \in X$;

$h(k, s)=0 \leftrightarrow$ for the least $n$ such that $\tau_{n-1} \in X\left\{e_{n-k}\right\}(s) \notin X$;

$h(k, s)=\uparrow \leftrightarrow$ there is no such $n$.

Then $h(k, s)$ is a function partial recursive in $X$, and when $X=H_{\lambda+n}$ then it is total with $\left\langle H_{\lambda+k} \mid 0 \leq k<n\right\rangle$ recursive in $H_{\lambda+n}$ as required. Q.E.D. Lemma 2.2

We seek to generalise the last observation on the definability of all $H_{\lambda+k}$ from $H_{\lambda+n}$ (for $k<n$ ) to all $\beta<\gamma<\Sigma$. We shall show (in Lemma 2.11 below) that:

The sequence $\left\langle H_{\gamma} \mid \gamma<\lambda\right\rangle$ is uniformly arithmetic in $H_{\lambda}$ for any limit $\lambda<\Sigma$.

Combining this then with (ii) of the last Lemma we shall have the uniform definability of $\left\langle H_{\gamma} \mid \gamma<\beta\right\rangle$ from $H_{\beta}$ for any $\beta<\Sigma$.

In our construction of the $L$ hierarchy we shall assume, somewhat non-standardly, that $L_{0}=V_{\omega}=\mathrm{HF}$ the hereditarily finite sets. This is just to make the numeration of our induction stages easier. $H_{1}$ contains all truths of arithmetic, and via a recursive function all truths of $\langle\mathrm{HF}, \epsilon\rangle$, hence it makes sense to start constructing 
the $L_{\alpha}$ 's with $L_{0}=\mathrm{HF}$. We express this well known fact concerning $\operatorname{Th}\left(\left\langle L_{0}, \epsilon,\right\rangle\right)$ and $T h(\langle\mathbb{N}, \cdots\rangle$,$) , that is H_{1}$, as:

Lemma 2.3 [Ackermann, $\mathrm{cf}$ [18] IV.3.22] There is a (1-1) recursive bijection $k$ : $\mathbb{N} \longrightarrow \mathbb{N}$ so that $\langle\mathrm{HF}, \epsilon\rangle \vDash \sigma \leftrightarrow\langle\mathbb{N},+, \times, \ldots\rangle \vDash k(\sigma)$. Consequently the theory $\mathrm{Th}\left(\left\langle L_{0}, \epsilon\right\rangle\right)$ is recursively isomorphic to $H_{1}$.

We shall make use of codes for wellfounded relations, whether they be wellorderings or the $\epsilon$-relation on (usually) transitive sets. If $\langle M, \epsilon\rangle$ is a structure, with $M$ a transitive countable set, we say that $E_{M} \subseteq \mathbb{N}$ is a code for $\langle M, \epsilon\rangle$ if there is an bijection $f: \mathbb{N} \leftrightarrow M$, and we have for $n, m \in \mathbb{N}$ that $f(n) \in f(m) \Longleftrightarrow\langle n, m\rangle \in E_{M}$. In short we have that $\left\langle\mathbb{N}, E_{M}\right\rangle$ is isomorphic to $\langle M, \epsilon\rangle$. A code for a wellorder is merely the special case when $M \in \mathrm{On}$. It is occasionally useful to have subsets of $\mathbb{N}$ rather than all of $\mathbb{N}$ coding wellorders. Such a subset is then the field of the coded wellorder.

We shall assume the reader is familiar with at least some of the details of the usual construction of the Gödel $L$ hierarchy. In particular the inductive construction of $\left\langle L_{\mu+1}, \epsilon\right\rangle$ from the structure $\left\langle L_{\mu}, \epsilon\right\rangle$. This is effected by looking at all subsets $X_{\varphi, \vec{y}}$ of $L_{\mu}$ definable using first order formulae in the language of set theory, $\varphi\left(v_{0}, y_{1}, \ldots, y_{k}\right)$ with parameters $\vec{y}=y_{i}$ from $L_{\mu}$. In our setting to follow, it is a fact that given the complete theory of the countable model $L_{\mu}-\operatorname{Th}\left(\left\langle L_{\mu}, \epsilon\right\rangle\right)$ - as a set of gödel numbers from $\mathbb{N}$, and given also any code for $\left\langle L_{\mu}, \epsilon\right\rangle$ in the sense above, call it $r_{\mu}$ say, one may by simple arithmetical operations on $r_{\mu}$ and the given theory, construct a code for $\left\langle L_{\mu+1}, \epsilon\right\rangle$.

Definition 2.4 (i) The $\Sigma_{n}$-Theory of $\left.\left\langle L_{\alpha}, \epsilon\right\rangle\right)$ will be abbreviated as $T_{\alpha}^{n}$; the complete theory will be denoted $T_{\alpha}$.

(ii) For $\operatorname{Lim}(\lambda)$, the Liminf theory at $\lambda$ is $\widehat{T}_{\lambda}={ }_{d f} \liminf _{\alpha \rightarrow \lambda} T_{\alpha}$.

We shall define two total recursive functions $l, g$, on which the construction will depend. The first of these will depend on the following lemma whose proof is deferred to Section 4.

Lemma 2.5 ( $H$-Limit Lemma) For limit $\lambda \leq \Sigma$ the $\Sigma_{2}$-theory of $\left\langle L_{\lambda}, \epsilon\right\rangle, T_{\lambda}^{2}$, is r.e. in $\widehat{T}_{\lambda}$. Moreover an index for this r.e. reduction is the same for all such $\lambda$.

Lemma 2.6 There is a total recursive function $l$, so that if $\lambda \leq \Sigma$ is any limit ordinal, and for any $e$, if (i) for all $\alpha<\lambda, T_{\alpha}=W_{e}^{H_{\alpha+1}}$ and (ii) for all limit $\mu<\lambda$ we have $W_{e}^{H_{\mu}}=\mathbb{N}$, then $T_{\lambda}=W_{l(e)}^{H_{\lambda+1}}$. 
Proof: Our assumptions in (i) and (ii) allow us to conclude that

$$
\liminf _{\alpha \rightarrow \lambda} W_{e}^{H_{\alpha}}=\liminf _{\alpha \rightarrow \lambda} W_{e}^{H_{\alpha+1}}=\widehat{T}_{\lambda} .
$$

Let a recursive (1-1) $\bar{g}$ be chosen (using $e$ ) with the property that $\bar{g}^{-1}$ " $H_{\alpha+1}=$ $W_{e}^{H_{\alpha}}$ (for all $\alpha$ ). The above equations translate then to:

$$
\bar{g}^{-1 ،} H_{\lambda}=\liminf _{\alpha \rightarrow \lambda} \bar{g}^{-1 “} H_{\alpha}=\liminf _{\alpha \rightarrow \lambda} \bar{g}^{-1 “} H_{\alpha+1}=\widehat{T}_{\lambda} .
$$

(The first equality holds, as the reader may check, as the liminf operation commutes with $\bar{g}^{-1}$; the middle equation holds because in turn $H_{\alpha}=\liminf _{\beta \rightarrow \alpha} H_{\beta}$ for $\operatorname{Lim}(\alpha), \alpha<\lambda$.) However $T_{\lambda}^{2}$ is uniformly r.e. in $\hat{T}_{\lambda}$ (by Lemma 2.5 and independently of $\lambda$ ). This implies that $T_{\lambda} \leq_{1}\left(H_{\lambda}\right)^{(\omega)}$. However there is a recursive and total $G_{1}$ witnessing that $\left(H_{\beta}\right)^{(\omega)}=G_{1}^{-1}$ “ $H_{\beta+1}$ for all $\beta$. Using this latter equation with $\beta=\lambda$ and putting it with the above, we can effectively find an index $l=l(e)$ with $T_{\lambda}=W_{l(e)}^{H_{\lambda+1}}$.

Q.E.D.

The second function $g$ will depend on:

Lemma 2.7 There is a recursive (1-1) function $G_{2}$ so that for $\operatorname{Succ}(\alpha), T_{\alpha}$ is (1-1) reducible to $\left(T_{\alpha-1}\right)^{(\omega)}$, i.e. so that: $T_{\alpha}=G_{2}^{-1}$ “' $\left(T_{\alpha-1}\right)^{(\omega)}$.

This will also be proven in Section 4 .

Lemma 2.8 There is a total recursive function $g$, so that if $\alpha<\Sigma$ is any successor ordinal, and for anye, if $T_{\alpha-1}=W_{e}^{H_{\alpha}}$, then $T_{\alpha}=W_{g(e)}^{H_{\alpha+1}}$.

Proof: Let $G_{1}$ be the fixed recursive functions from above so that for any $\alpha<$ $\Sigma H_{\alpha}^{(\omega)}=G_{1}^{-1}$ “ $H_{\alpha+1}$. For any $e$ let $Z(e)=W_{e}^{H_{\alpha}}$. $Z(e)$ is thus a possible candidate for $T_{\alpha-1}$, depending on the choice of $e$. Now we have $T_{\alpha} \leq_{1}\left(T_{\alpha-1}\right)^{(\omega)}$, via the fixed function $G_{2}$ of Lemma 2.7. Thus $T_{\alpha}=G_{2}^{-1}$ “ $\left(T_{\alpha-1}\right)^{(\omega)}$.

Let $H_{e}$ be a fixed function depending on $e$ which witnesses that $Z(e)^{(\omega)} \leq_{1}$ $H_{\alpha}^{(\omega)}$. Hence $Z(e)^{(\omega)}=H_{e}^{-1}$ " $H_{\alpha}^{(\omega)}$. Let $G_{e}$ be the (1-1) function $H_{e} \circ G_{2}$. Then in case $Z(e)=T_{\alpha-1}$, we shall have that $T_{\alpha}=G_{e}^{-1 \text { " }} H_{\alpha}^{(\omega)}$. Finally let $g(e)$ be an index so that $W_{g(e)}^{H_{\alpha+1}}=\left(G_{1} \circ G_{e}\right)^{-1}$ “ $H_{\alpha+1}$. Again if $Z(e)=T_{\alpha-1}$, then $T_{\alpha}=W_{g(e)}^{H_{\alpha+1}}$.

Q.E.D. Lemma 2.8

Lemma 2.9 There is an index $e_{0}$ and thus a (1-1) recursive function $G_{L}$ so that for all $\alpha<\Sigma$ : (i) $W_{e_{0}}^{H_{\alpha+1}}=T_{\alpha}$; (ii) $T_{\alpha}=G_{L}^{-1}$ " $H_{\alpha+2}$. 
Proof: We proceed to define $f(e, n)$ a partial function recursive in an arbitrary $X$. The indices $g(e), l(e)$ use the functions $g, k, l$ from the lemmas 2.8, 2.3, 2.6 above.

$$
\begin{array}{cl}
f(e, n)=1 \quad & \text { if }\ulcorner(\dot{T}\ulcorner 0=0\urcorner)\urcorner \notin X \wedge n \in k^{-1 “ X} ; \\
& \text { or if } \tau_{0} \in X \wedge\{l(e)\}^{X}(n) \downarrow ; \\
& \text { or if neither } L_{0} \operatorname{nor} \neg L_{0} \text { is in } X ; \\
& \text { or if } \tau_{0} \notin X \wedge\left(L_{0} \in X \vee \neg L_{0} \in X\right) \wedge\{g(e)\}^{X}(n) \downarrow . \\
\text { In all other cases } f(e, n) \uparrow .
\end{array}
$$

By the Recursion Theorem there is $e_{0}$ so that for any $X,\left\{e_{0}\right\}^{X}(n)=f\left(e_{0}, n\right)$.

Claim: $\forall \alpha<\Sigma W_{e_{0}}^{H_{\alpha+1}}=T_{\alpha}$. For $\operatorname{Lim}(\alpha)$ we have $\liminf _{\beta \rightarrow \alpha} W_{e}^{H_{\beta}}=\hat{T}_{\alpha}$.

Proof: By induction on $\alpha$, including additionally the claim that for $\operatorname{Lim}(\alpha)$ that $W_{e_{0}}^{H_{\alpha}}=\mathbb{N}$. For $\alpha=0$ this is trivial. If true for $\beta$ where $\alpha=\beta+1$, then let $X=$ $H_{\alpha+1}$. Then $\tau_{0} \notin X \wedge\left(L_{0} \in X \vee \neg L_{0} \in X\right)$ and thus $W_{e_{0}}^{H_{\alpha+1}}=\operatorname{dom}\left(\left\{g\left(e_{0}\right)\right\}^{X}\right)=T_{\alpha}$ as required. If now true for $\beta<\alpha$ where $\operatorname{Lim}(\alpha)$ then we have neither $L_{0}$ nor $\neg L_{0}$ is in $X=H_{\alpha}$, and thus $W_{e_{0}}^{H_{\alpha}}=\mathbb{N}$.

Still with $\operatorname{Lim}(\alpha)$, if $X=H_{\alpha+1}$, as $\tau_{0} \in X, W_{e_{0}}^{H_{\alpha+1}}=\operatorname{dom}\left(\left\{l\left(e_{0}\right)\right\}^{X}\right)=T_{\alpha}$, the latter equality by our fulfillment of the conditions to apply Lemma 2.6.

Q.E.D. Claim.

The Claim proves (i) of course, and (ii) then is immediate (since for any $e$, $W_{e}^{H_{\alpha+1}}$ is (1-1) reducible to $H_{\alpha+1}^{(\omega)} \leq_{1} H_{\alpha+2}$, the latter via $G_{1}$ as remarked above).

Q.E.D. Lemma 2.9

We shall make use of the following corollary to the proof of Lemma 2.5 (also proven in Section 4):

Corollary 2.10 (Wellordering Lemma) (cf. [8]) There is a single recursive function $G: \mathbb{N} \times \mathbb{N} \longrightarrow \mathbb{N}$, so that for any limit ordinal $\beta$, if we set

$$
w_{\beta}=\left\{\prec u, v>\in \mathbb{N} \mid \exists i \in \mathbb{N} G(i, \prec u, v>) \in H_{\beta}\right\}
$$

then $w_{\beta}$ codes a well ordering $<_{\beta}$ of $\mathbb{N}$ of type $\beta$.

\section{Proof of Theorem 1.1.}

Let $f_{0}$ be from Lemma 2.2 (i), and $G$ the function just mentioned in the Corollary 2.10. For a subset $A \subseteq \mathbb{N} \times \mathbb{N}$, let $(A)_{1}=_{\text {df }}\{m \mid \exists n\langle n, m\rangle \in A\}$. Suppose $\beta \leq \gamma$ and let $\beta=\omega \cdot k+l, \gamma=\omega \cdot k^{\prime}+l^{\prime}$ for some $k \leq k^{\prime}$. Suppose we assumed $H_{\gamma} \subseteq H_{\beta}$. Then by Lemma 2.1 (ii) we must have $l=l^{\prime}$. However we also have that $\left(G^{-1 "} f_{0}^{-1 "} H_{\gamma}\right)_{1} \subseteq\left(G^{-1 "} f_{0}^{-1 \text { “ }} H_{\beta}\right)_{1}$ (if $l>0$ ), and $\left(G^{-1 “} H_{\gamma}\right)_{1} \subseteq\left(G^{-1 \text { " }} H_{\beta}\right)_{1}$ (if $l=0$ ). 
Either alternative implies that $w_{\omega . k^{\prime}}$ is a wellorder of type $\omega . k^{\prime}$ contained in $w_{\omega . k}$ a wellorder of type $\omega . k$. Thus $k=k^{\prime}$. Thus $\beta=\gamma$. This completes the theorem.

Q.E.D. Theorem 1.1

(Moreover this last proof is also the basis of the "non-wellfounded" version mentioned in [24], if, for example, we took $\beta$ an ordinal and $c$ likewise an ordinal in the illfounded part of the ordering, (with largest limit ordinals less than them of $\beta^{\prime}$ and $c^{\prime}$ respectively then we'd have that $w_{\beta^{\prime}}$ would contain $w_{c^{\prime}}$ as a suborder - but this is also absurd, as $w_{c^{\prime}}$ is illfounded).

We now turn to our claims that the whole sequence up to a stage is recoverable from that stage: Lemma 1.3(i). We first consider limit ordinals $\lambda$.

Lemma 2.11 Let $\lambda<\Sigma$ be a limit ordinal. Then $\left\langle H_{\alpha} \mid \alpha<\lambda\right\rangle$ is uniformly definable over $L_{\lambda}$. Moreover a code for this sequence can be found uniformly arithmetically in $H_{\lambda}$.

Proof: From above we have a wellorder, $<_{\lambda}$ from the relation $w_{\lambda}$, of order type $\lambda$ that is uniformly $\Sigma_{2}^{L_{\lambda}}$. That is, there is a $\Sigma_{2}$ definition of a binary relation, that works over any limit $\lambda<\Sigma$ to define $w_{\lambda}(n, m)$, a wellordering of that length. (To see that it is $\Sigma_{2}$ over $L_{\lambda}$, recall that $H_{\lambda}$ itself is $\Sigma_{2}$ over $L_{\lambda}$ and $w_{\lambda}$ is $\left(G^{-1 \text { “ }} H_{\lambda}\right)_{1}$.) Consequently we may define a code for the iteration of our revision sequence along this ordering:

$$
\begin{aligned}
& \mathscr{H}_{\lambda}(k, m) \leftrightarrow\left\langle L_{\lambda}, \in\right\rangle \vDash " \exists f \exists n\left[n \in \operatorname{Field}\left(w_{\lambda}\right) \wedge \operatorname{Fun}(f) \wedge \operatorname{dom}(f)=\left\{p \mid w_{\lambda}(p, n)\right\} \wedge\right. \\
& \wedge \forall u( \\
& \quad\left(u \text { is }<_{\lambda} \text {-least } \longrightarrow f(u)=\varnothing\right) \wedge \\
& \quad\left(u \text { a }<_{\lambda} \text {-successor of } v \longrightarrow f(u)=\{\ulcorner\sigma\urcorner \mid\langle\mathbb{N},+, \times, \ldots, f(v)\rangle \models \sigma\}\right) \wedge \\
& \quad\left(u \text { a }<<_{\lambda} \text {-limit } \longrightarrow f(u)=\liminf _{\nu<_{\lambda} u} f(v)\right) \wedge \\
& \quad \wedge k \in f(m)] .
\end{aligned}
$$

The relation $\mathscr{H}_{\lambda}(k, m)$ codes $\left\langle H_{\alpha} \mid \alpha<\lambda\right\rangle$ : if $\alpha<\lambda$ and $m$ is such that $|m|_{<_{\lambda}}=$ $\alpha$ then $H_{\alpha}=\left\{k \mid \mathscr{H}_{\lambda}(k, m)\right\}$. Due to the uniformity in the definition of $w_{\lambda}$ this $\left(\Sigma_{4}^{L_{\lambda}}\right)$ definition of $\mathscr{H}_{\lambda}$ is independent of $\lambda$.

For the last sentence of the lemma: since $T_{\lambda}^{2}$ is r.e. in $H_{\lambda}$, and $\mathscr{H}_{\lambda}$ is arithmetical in $T_{\lambda}^{2}$, we have that $\mathscr{H}_{\lambda}$ is then arithmetical in $H_{\lambda}$, again all uniformly.

Q.E.D. Lemma 2.11

Thus for such $\lambda$ we have a way not only of defining simply a wellorder of type $\lambda$ from $H_{\lambda}$, but we have a single method for recovering the whole prior sequence $\left\langle H_{\gamma} \mid \gamma<\lambda\right\rangle$ from knowledge of $H_{\lambda}$. We now marry the above Lemma 
with Lemma 2.2.

\section{Proof of Lemma 1.3 (i) for the $H$ sets:}

For $\beta$ a limit the last lemma shows us how to decode the whole sequence up to $\beta$ from $H_{\beta}$ in a way that is uniform for all such limits $\beta<\Sigma$. We have also seen in Lemma 2.2 that if $\beta=\lambda+k$ where $\lambda$ is the largest limit ordinal less than $\beta$ how to recover $k$, and the sets $H_{\lambda+k^{\prime}}$ for $k^{\prime}<k$. Since from $H_{\lambda}$ we may define $\left\langle H_{\alpha} \mid \alpha<\lambda\right\rangle$, we may recover a code for this sequence in a recursive way from $H_{\beta}$. Finally we may glue together this code with those of the finitely many sets $H_{\lambda+k^{\prime}}$ for $k^{\prime}<k$, (taking care to do this in a way that only depends on $k$ ) to get a code for $\left\langle H_{\alpha} \mid \alpha<\beta\right\rangle$ arithmetically from $H_{\beta}$. Q.E.D. Lemma 1.3 (i) for the $H$-sets.

\section{The Fieldian $F_{\gamma}$ sets and determinateness hierarchies}

In this section we consider how the above needs modifying to obtain the same results for the Fieldian hierarchy. In the second part we see how to define determinateness path hierarchies.

\subsection{The F-hierarchy}

The point of the definition of our $F_{\beta}$, is that it encapsulates the semantic values of the sentences $A$ at stages in Field's construction prior to $\beta$ : if $\beta=\delta+1$ then $F_{\beta}$ encapsulates the semantic values of all $|A|_{\delta, \Omega}$ at the end of the $\delta$ 'th round through an inspection to see if it contains $\langle\top \longrightarrow A\urcorner, 1\rangle$ or $\langle\ulcorner A \longrightarrow \perp\urcorner, 1\rangle$; or if $\operatorname{Lim}(\beta)$ then the values of those $T \longrightarrow A$ etc. that stabilize. Given then $F_{\beta}$ we have the complete distribution of semantic values needed to proceed to calulating the $\beta$ 'th round of a fixed point. This fixed point is built up in a standard fashion for a three valued Strong Kleene logic. Thus, for example, the first stage builds up semantic values $|\operatorname{Tr}(\ulcorner A\urcorner)|_{\beta, 1}$ equalling $1,0, \frac{1}{2}$ depending on the set $F_{\beta}$ alone. (Field resets all values $|\operatorname{Tr}(\ulcorner A\urcorner)|_{\beta, 0}$ to $\frac{1}{2}$ at the start of each major stage.) Thus $|\operatorname{Tr}(\ulcorner A \longrightarrow B\urcorner)|_{\beta, 1}$ equals 1,0, $\frac{1}{2}$ depending on whether $\langle\ulcorner A \longrightarrow$ $B\urcorner, 1\rangle,\langle\ulcorner A \longrightarrow B\urcorner, 0\rangle$ or neither is in $F_{\beta}$. Consequently any arithmetic statement $\Phi_{0}$ true in the structure $\left\langle\mathbb{N}, F_{\beta}\right\rangle$ is then, apart from some inessential syntactic coding, a true arithmetic statement $\Phi$ in the basic values $|\operatorname{Tr}(\ulcorner A \longrightarrow B\urcorner)|_{\beta, 1}$; i.e. $|\operatorname{Tr}(\Phi)|_{\beta, 2}=1$ and hence $|\operatorname{Tr}(\Phi)|_{\beta}=1$. (This corresponds, when building up the first minimal Strong Kleene fixed point over arithmetic, to having the extensions of Tr initially empty, and then all basic arithmetic truths (in the Tr-free part of the language) are then immediately placed into the extension of $\mathrm{Tr}$ at the very next stage, and so end up in the fixed point.) In short, it suffices to consider the sequence of sets $F_{\beta}$ when thinking how the ultimate truths in the model are built 
up, and we shall not always distinguish $\Phi_{0}$ from the coresponding implicit $\Phi$ in the above.

We let $\left\langle\tau_{\iota} \mid \iota \leq \Sigma\right\rangle$ enumerate in ascending order $\mathrm{ADM}^{*}$, the closed and unbounded sequence of admissible ordinals together with their limit points, below $\Sigma$. We set $\tau_{0}=0$, and thus $\tau_{1}=\omega_{1}^{\mathrm{ck}}$. It can be shown that $\tau_{\zeta}=\zeta$ and $\tau_{\Sigma}=\Sigma$. Note: not every limit of admissible ordinals is admissible.

Essentially we want to rerun the argument for the $H$-sets but for the $F$-sets: the difference is that at each stage instead of using definable sets of the previous level to go one level up in the $L$ hierarchy, from $L_{\alpha}$ to $L_{\alpha+1}$ when going from $H_{\alpha}$ to $H_{\alpha+1}$, we take a whole admissible jump up: from $L_{\tau_{\alpha}}$ to $L_{\tau_{\alpha+1}}$ when going from $F_{\alpha}$ to $F_{\alpha+1}$.

Just as we did for the $H$ sets we make some simple observations about successor steps.

Lemma 3.1 (i) There is an effective procedure for testing $F_{\beta}$ to determine if $\beta$ is a limit ordinal.

(ii) For $\omega>n>0$ there is a sentence $\tau_{n}$ so that

$\forall \beta\left[\tau_{n} \in F_{\beta} \leftrightarrow \exists \lambda(\operatorname{Lim}(\lambda) \wedge(\beta=\lambda+n))\right]$.

Proof: (i) Let $K$ be the Curry sentence equivalent to $T(\ulcorner K\urcorner) \longrightarrow \perp$. Then $\operatorname{Lim}(\beta) \leftrightarrow|K|_{\beta}=\frac{1}{2} \leftrightarrow\langle T(\ulcorner K\urcorner) \longrightarrow \perp, 1\rangle,\langle T(\ulcorner K\urcorner) \longrightarrow \perp, 0\rangle \notin F_{\beta}$.

For (ii): $|K|_{\lambda+n}$ alternates value between 0 and 1 for $0<n<\omega$; suppose $n>0$.

$n=1 \leftrightarrow\langle(K \wedge \neg K) \longrightarrow \perp, 1\rangle \in F_{\lambda+n}$. So we may take $\tau_{1}$ to be $(K \wedge \neg K) \longrightarrow \perp$.

$n=2 \leftrightarrow\left\langle\top \longrightarrow \tau_{1}, 1\right\rangle \in F_{\lambda+n}$.

$n=3 \leftrightarrow\left\langle\top \longrightarrow\left(\top \longrightarrow \tau_{1}\right), 1\right\rangle \in F_{\lambda+n}$ and so forth adding " $T \longrightarrow$ " for each extra increase in $n$.

Q.E.D.

Above we have indicated how the $F_{\beta}$ sets fit into Field's description of his model, and indeed the sets encapsulate everything we get to know about the model and the set of ultimate truths, which we shall denote $F_{\zeta}=F_{\Delta_{0}}$, and we obtain that $\|A\|$ equals $1,0, \frac{1}{2}$ depending on whether $\langle T \longrightarrow A, 1\rangle,\langle A \longrightarrow \perp, 1\rangle$ or neither, is in $F_{\zeta}$.

In the context of the $F$-hierarchy, $F_{\alpha+1}$ is a complete $\Pi_{1}^{1, F_{\alpha}}$ set of integers, essentially by considerations originating with Kripke ( $c f$. [22] Prop. 2.5) and because of this we can recursively recover the complete $\Sigma_{1}$-Theory of $\left\langle L_{\tau_{\alpha+1}}\left[F_{\alpha}\right], \epsilon\right.$ , $\left.F_{\alpha}\right\rangle$ from $F_{\alpha+1}$ ( $c f$. [22] Prop. 2.6). The method of recovering this theory does not depend on $\alpha$. We shall use the notation that $j_{K}(F)=G$ where $G$ is the set of ordered pairs $\langle A, i\rangle$ of sentences that come True (for $i=1$ ) (or False for $i=0$ ) in the minimal Strong Kleene fixed point over the starting value distribution coded into $F$. Hence for each $\alpha: j_{K}\left(F_{\alpha}\right)$ can be read off from $F_{\alpha+1}$ : 
$\left.\langle A, 1\rangle \in j_{K}\left(F_{\alpha}\right) \leftrightarrow\langle\top \longrightarrow A\urcorner, 1\right\rangle \in F_{\alpha+1}$ (and similarly for $\langle A, 0\rangle$ m.m.). It is this 'Strong Kleene jump' that produces for us Field's hierarchy. ${ }^{2}$

Of course $F_{\alpha+1}$ gives us the complete $\Sigma_{2}$ theory of $\left\langle L_{\tau_{\alpha+1}}\left[F_{\alpha}\right], \in, F_{\alpha}\right\rangle$ as well: the latter is recursive in the Turing jump of $F_{\alpha+1}: F_{\alpha+1}^{\prime}$. In our terminology from above, from this we shall also have that $T_{\tau_{\alpha+1}}^{2} \leq_{1} F_{\alpha+1}^{\prime}$ in a uniform fashion. This is stated as (i) of the next Lemma which is proven as part of Lemma 2.2 from [22]. (Note in [22] $F_{l}$ here is called essentially $C_{l}$ there.)

Lemma 3.2 For $\iota<\Sigma$ (i) $T_{\tau_{t+1}}^{2} \leq_{1} F_{\iota+1}^{\prime}$ uniformly in $\iota$.

(ii) $\operatorname{Lim}(\iota) \wedge L_{\tau_{\iota}}=\Sigma_{1}$-Separation $\longrightarrow T_{\tau_{\iota}}^{2} \leq_{1} F_{\iota}$, uniformly in $\iota$.

For the limit case, in [22] Lemma 2.2, this stronger reduction in (ii) of $T_{\tau_{\lambda}}^{2} \leq_{1}$ $F_{\lambda}$ was shown only uniformly for those $\lambda$ with $L_{\tau_{\lambda}} \vDash \Sigma_{1}$-Separation: this was sufficient for our arguments at that time. However we had missed the uniformity over all $\lambda<\Sigma$ that can be obtained from the $F$-Limit Lemma 3.3 below. This gives us then for any limit $\lambda$ that we have $T_{\tau_{\lambda}}^{2}$ is uniformly r.e. in $F_{\lambda}$, (so a weaker condition, but a weaker conclusion) and this is just as we had for the $H$-sets. We shall need the uniformity to get the 'uniform recoverability' property.

The limit level procedures are in the essential mathematical respects the same: liminf's are taken, and the Limit Lemma and Wellordering Lemma have the following unchanged form (and proofs).

Lemma 3.3 ( $F$-Limit Lemma) For a limit $\lambda \leq \Sigma$ the $\Sigma_{2}$ theory of $\left\langle L_{\tau_{\lambda}}, \epsilon\right\rangle, T_{\tau_{\lambda}}^{2}$, is r.e. in $F_{\lambda}$. Moreover an index for this r.e. reduction is uniform in $\lambda$. 4):

Hence $T_{\tau_{\lambda}}^{2}$ is $\Sigma_{1}\left(\left\langle\mathbb{N}, F_{\lambda}\right\rangle\right)$. Just as for the $H$-hierarchy we shall have (Section

Corollary 3.4 (Wellordering Lemma) (cf. [8]) There is a single recursive function $G_{F}: \mathbb{N} \times \mathbb{N} \longrightarrow \mathbb{N}$, so that for any limit ordinal $\beta<\lambda$, if we set

$$
w_{\tau_{\beta}}=\left\{\prec u, v>\in \mathbb{N} \mid \exists i \in \mathbb{N} G(i, \prec u, v>) \in F_{\beta}\right\}
$$

then $w_{\tau_{\beta}}$ codes a well ordering of $\mathbb{N}$ of type $\beta$.

Lemma 3.5 (i) There is a (1-1) total recursive function $f_{0 F}$ so that for any limit $\lambda$ and any $n<\omega$, then $F_{\lambda}=f_{0 F}^{-1}$ " $F_{\lambda+n+1}$.

(ii) Moreover the sequence $\left\langle F_{\lambda+k} \mid 0 \leq k<n\right\rangle$ is uniformly recursive in $F_{\lambda+n}$ for any such $\lambda$ and $n \in \mathbb{N}$.

\footnotetext{
${ }^{2}$ The reader may notice that in [22] we used the slightly different sets $C_{\alpha}$ rather than $F_{\alpha}$; there $C_{\alpha}$ contained only pairs of the form $\left.\langle\top \longrightarrow A\urcorner, 1\right\rangle,\langle\ulcorner A \longrightarrow \perp\urcorner, 1\rangle$; so an effective subset of what we are calling $F_{\alpha}$ here; but clearly this does not alters the results.
} 
Proof: Similar to Lemma 2.2 and left to the reader.

Q.E.D. Lemma 3.5.

\section{Proof of Theorem 1.2(Non-decreasing)}

Employ the same argument as for the $H$ sets, using the functions $F$ from Lemma 3.5 and $G_{F}$ from Lemma 3.4.

Q.E.D. Theorem 1.2

\section{Proof of Lemma 1.3}

This will follow from the next Lemma.

Lemma 3.6 Let $\gamma<\Sigma$. Then $\left\langle F_{\alpha} \mid \alpha<\gamma\right\rangle$ is uniformly definable over $L_{\tau_{\gamma}}$. Moreover for limit $\gamma$ a code for this sequence can be found uniformly arithmetically in $F_{\gamma}$.

Proof: One should first note that $\mathrm{ADM}^{*} \cap \tau_{\gamma}$ is uniformly $\Delta_{1}^{L_{\tau_{\gamma}}}$ and its order type is of course $\gamma \leq \tau_{\gamma}$.

Using Lemma 4.1, uniformly in $\gamma$, there is a $\Sigma_{2}^{L_{\tau_{\gamma}}}$ definable partial map $g_{\tau_{\gamma}}$ of a subset of $\omega$ onto $L_{\tau_{\gamma}}$. We thus again have a wellorder, $<_{\tau_{\gamma}}$ from the relation $w_{\tau_{\gamma}}$, of order type $\gamma$ that is uniformly $\Sigma_{2}^{L_{\tau_{\gamma}}}$. That is, there is a $\Sigma_{2}$ definition of a binary relation, that over any $L_{\tau_{\gamma}}$ for $\gamma<\Sigma$, defines $w_{\tau_{\gamma}}(n, m)$, a wellordering of that length. Consequently we may define a code for the iteration of our revision sequence along this ordering:

(1) $\mathscr{F}_{\gamma}(k, m) \leftrightarrow \exists f \exists n\left[n \in \operatorname{Field}\left(w_{\tau_{\gamma}}\right) \wedge \operatorname{Fun}(f) \wedge \operatorname{dom}(f)=\left\{m \mid w_{\tau_{\gamma}}(m, n)\right\} \wedge\right.$ $\forall u($

$\left(u\right.$ is $<_{\tau_{\gamma}}$-least $\left.\longrightarrow f(u)=\varnothing\right) \wedge$

$\left(u \mathrm{a}<{ }_{\tau_{\gamma}}\right.$-successor of $\left.v \longrightarrow f(u)=j_{K}\left(F_{f(v)}\right)\right) \wedge$

$\left(u \mathrm{a}<_{\tau_{\gamma}}-\operatorname{limit} \longrightarrow f(u)=\liminf _{\nu<_{\tau_{\gamma}} u} f(v)\right) \wedge$

$\wedge k \in f(m))]$.

In the above we have used the function " $j_{K}(F)=G$ " which proceeds from a set of semantic values to its "Fieldian jump". If $\gamma$ is a limit, this function is total on such semantic sets and is moreover $\Delta_{1}^{L_{\tau_{\gamma}}}$ definable. (To determine $G$ from $F$ one needs only to go to the least transitive admissible set containing $F$, and the values of $G$ are $\Sigma_{1}$-definable over it; any $F$ we have is in some $L_{\tau_{\delta}}$ and then $j_{K}(F)$ is uniformly definable over $L_{\tau_{\delta+1}}$.) However even if $\gamma$ is, say $\lambda+k+1$ with $\lambda$ the largest limit below $\gamma$, one may apply the same function $j_{K}$ to the sets $F_{\lambda}, F_{\lambda+1}=j_{K}\left(F_{\lambda}\right), \ldots, F_{\lambda+k}=j_{K}\left(F_{\lambda+k-1}\right)$, and again this is $\Delta_{1}^{L_{\tau_{\gamma}}}$ definable. The 
length of the domain of any such function $f$ occurring in (1) above can thus be any $\gamma^{\prime}<\gamma$.

The relation $\mathscr{F}_{\gamma}(k, m)$ codes $\left\langle F_{\alpha} \mid \alpha<\gamma\right\rangle$ : if $\alpha<\gamma$ and $m$ is such that $|m|_{<_{\tau_{\gamma}}}=$ $\alpha$ then $F_{\alpha}=\left\{k \mid \mathscr{F}_{\gamma}(k, m)\right\}$. Due to the uniformity in the definition of $w_{\tau_{\gamma}}$, and of $j_{K}$, the $\left(\Sigma_{4}^{L_{\tau_{\gamma}}}\right)$ definition of $\mathscr{F}_{\gamma}$ is independent of $\gamma$. $\mathscr{F}_{\gamma}(k, m)$ is thus, uniformly, arithmetical in $T_{\tau_{\gamma}}^{2}$.

The last sentence of the lemma follows since $T_{\tau_{\gamma}}^{2}$ is uniformly r.e. in $F_{\gamma}$ if $\gamma$ is any limit.

Q.E.D. Lemma 3.6/

One may conclude the proof of Lemma 1.3 (ii) from the last lemma in exactly the same way 1.3(i) was concluded from Lemma 2.11. Q.E.D. Lemma 1.3 (ii) for the $F$.

\subsection{Determinateness hierarchies}

We address the problem of the length of possible determinateness path hierarchies as outlined in Field's book [5], $c f$. also [6] where this is also discussed.

We use the above analysis to derive the 'stabilizing' formulae $P_{<}$and $P_{\leq}$that we have discussed in [25] and appear in the lemmata above.

Proof of Lemma 1.5: We have seen that there is a single arithmetical formula $\Phi$ that defines over any $\left\langle\mathbb{N}, F_{\beta}\right\rangle$ for $(\beta<\Sigma)$ a wellorder of type $\beta$ together with the associated previous $F$-sets $\left\langle F_{\alpha} \mid \alpha<\beta\right\rangle$. In particular it means that many things that we might express in a first order way about the sequence $\left\langle F_{\gamma}\right| \gamma<$ $\beta\rangle$, for example whether a particular sentence $A$ is stably 0 , is then translatable into a standard two valued arithmetic statement in the language of arithmetic augmented by a symbol for $F_{\beta}$, that is, or is not, true in $\left\langle\mathbb{N}, F_{\beta}\right\rangle$. We exploit this to prove the Lemma.

Let $X(x)$ be: " $\forall \alpha \exists \beta>\alpha|x|_{\beta} \neq|x|_{\alpha}$." This expresses that $x$ has an unstable semantic value. Let $\tilde{A}_{X}\left(\nu_{0}\right)$ be the arithmetical equivalent of this using this translation into the ordinary language of arithmetic, effected in such a way so that $\left\{\ulcorner B\urcorner \mid\left\langle\mathbb{N}, F_{\beta}\right\rangle \models \tilde{A}_{X}(\ulcorner B\urcorner)\right\}$ is the set of sentences unstable below $\beta$.

Recall that $F_{\beta}$ is the set of ordered pairs $\langle\ulcorner A\urcorner, j\rangle$ with $A$ a conditional, and $j<\epsilon 2$ indicating whether $|A|_{\beta, 0}=j$. Hence, still for such $A$, we have, for an atomic clause,

$$
\text { “ }\langle\ulcorner A\urcorner, 1\rangle \in F_{\beta} ” \leftrightarrow|\ulcorner A\urcorner|_{\beta, 0}=1 \leftrightarrow|\operatorname{Tr}(\ulcorner A\urcorner)|_{\beta, 1}=1
$$

and similarly,

$$
\text { “ }\ulcorner\ulcorner\urcorner, 0\rangle \in F_{\beta} ” \leftrightarrow|\ulcorner A\urcorner|_{\beta, 0}=0 \leftrightarrow|\operatorname{Tr}(\ulcorner A\urcorner)|_{\beta, 1}=0
$$


with $|\operatorname{Tr}(\ulcorner A\urcorner)|_{\beta, 1}=\frac{1}{2}$ otherwise.

Hence our two-valued arithmetic statement $\widetilde{A}_{X}$ about $F_{\beta}$ is translatable in turn to a similar two valued statement, call it $A_{X}$, but now in the language $\mathscr{L}^{+}$, about the truth sets of conditionals $\operatorname{Tr}(\ulcorner A\urcorner)$ at stage $\beta, 1$. This holds in a similar fashion for any arithmetical formula concerning $\left\langle\mathbb{N}, F_{\beta}\right\rangle$.

Note that $\left\|A_{X}(x)\right\|=0 \leftrightarrow \rho(x) \downarrow$. Note also that if $\beta=\delta+1$ then trivially $\left\langle\mathbb{N}, F_{\beta}\right\rangle \vDash \neg \tilde{A}_{X}(n)$ for any sentence with code $n$. However if $\operatorname{Lim}(\beta)$ then $\left\langle\mathbb{N}, F_{\beta}\right\rangle=\tilde{A}_{X}(n)$ is possible if $n$ is unstable below $\beta$. In that case $\left|A_{X}(n)\right|_{\beta, \Omega}=$ $\left|\mathrm{T} \longrightarrow A_{X}(n)\right|_{\beta+1}=1$. We may thus conclude that

$$
\|x\|=1 \backslash 2 \leftrightarrow \rho(x) \uparrow \leftrightarrow\left\|\top \longrightarrow A_{X}(x)\right\|=1 \backslash 2 \leftrightarrow\left\|A_{X}(x)\right\|=1 \backslash 2 .
$$

And

$$
\rho(x) \downarrow \leftrightarrow\left\|\top \longrightarrow A_{X}(x)\right\|=0 \leftrightarrow\left\|A_{X}(x)\right\|=0 .
$$

Let $\Upsilon(x, y)$ abbreviate

"if $\alpha_{x}, \alpha_{y}$ are least so that $\forall \beta \geq \alpha_{x} \forall \gamma \geq \alpha_{y}\left(|x|_{\beta}=|x|_{\alpha_{x}} \wedge|y|_{\gamma}=|y|_{\alpha_{y}}\right)$ then $\alpha_{x} \leq \alpha_{y}$.

Now let $\Psi_{\leq}(x, y)$ be: $X(x) \vee[\neg X(x) \wedge \neg X(y) \wedge \Upsilon(x, y)]$.

Let $\tilde{A}_{\Psi_{\leq}}\left(v_{0}, v_{1}\right)$ be the translation of $\Psi_{\leq}(x, y)$ and let $P_{\leq}(x, y) \equiv A \Psi_{\leq}(x, y)$ be the corresponding $\mathscr{L}^{+}$formula. We check that $P_{\leq}$is as demanded by the Lemma.

Claim:

$$
\begin{aligned}
\left\|P_{\leq}(\ulcorner A\urcorner,\ulcorner B\urcorner)\right\| & =1 \text { iff } \rho(A) \downarrow, \rho(B) \downarrow \wedge \rho(A) \leq \rho(B) \\
& =0 \text { iff } \rho(A) \downarrow, \rho(B) \downarrow \wedge \rho(A)>\rho(B) \\
& =\frac{1}{2} \text { otherwise. }
\end{aligned}
$$

Proof of Claim: First set $x=\ulcorner A\urcorner$ and $y=\ulcorner B\urcorner$. Note that the first line is straightforward.

$$
\left\|P_{\leq}(x, y)\right\|=1 \leftrightarrow\left\|A_{\Psi_{\leq}}(x, y)\right\|=1 \leftrightarrow\left\|A_{X}(x)\right\|=\left\|A_{X}(y)\right\|=0 \wedge \rho(x) \leq \rho(y) .
$$

Towards the second line, suppose $\left\|P_{\leq}(x, y)\right\|=0$. Then $x$ is stable since otherwise $\|x\|=\frac{1}{2} \leftrightarrow\left\|A_{X}(x)\right\|=\frac{1}{2}$ which implies that $\left\|P_{\leq}(x, y)\right\| \geq \frac{1}{2}$. If $\rho(y) \uparrow$ then $\left\|A_{X}(y)\right\|=\frac{1}{2}$. As $\neg \tilde{A}_{X}(y)$ is true for all successor $\gamma=\delta+1$ over $\left\langle\mathbb{N}, F_{\gamma}\right\rangle$, by our supposition that $P_{\leq}(x, y)=0$ we must have for each (successor) $\gamma$ that “ $\neg X(y) \wedge$ 
$\Upsilon(x, y)$ " is false over such $\left\langle\mathbb{N}, F_{\gamma}\right\rangle$. However as $\neg \tilde{A}_{X}(y)$ is true this can only be false over all sufficiently large successor $\gamma$ if $\alpha_{y}$ is defined over $\left\langle\mathbb{N}, F_{\gamma}\right\rangle$ and is less than $\alpha_{x}$ as there defined. But $\rho(x) \downarrow$ so $\alpha_{x}$ is defined as the same ordinal for all sufficiently large successor $\gamma$. Then we must have $\rho(y) \downarrow \wedge \rho(y)<\rho(x)$.

The converse is straightforward. And hence $\left\|P_{\leq}(x, y)\right\|=\frac{1}{2}$ in the remaining cases. The definition of $P_{<}(x, y)$ is done analogously. QED Lemma 1.5.

Proof of Lemma 1.6 It suffices to show that $\zeta_{0}={ }_{\mathrm{df}}$ ot $(<)=\zeta$. Note first that $\zeta_{0} \leq \zeta$ since by definition of $\Delta_{0}=\zeta$ it is the least acceptable point, i.e. any sentence that is going to stabilize will do so by stage $\zeta$. We show that $\zeta_{0} \geq \zeta$.

For $\beta \in$ On let $S_{\beta}^{1}=_{\mathrm{df}}\left\{\alpha \mid L_{\alpha} \prec_{\Sigma_{1}} L_{\beta}\right\}$. It is a standard fact, and easily seen, that if $\alpha \leq \beta$ is a limit point of $S_{\beta}^{1}$ then $L_{\alpha} \vDash \Sigma_{1}$-Separation.

By the reflection property that defines $\zeta$ as the least such that there is $\Sigma>\zeta$ with $L_{\zeta} \prec_{2} L_{\Sigma}$, one may show that $S={ }_{\mathrm{df}} S_{\zeta}^{1}$ is unbounded in $\zeta$ and has order type $\zeta$. (This is essentially because $L_{\zeta} \vDash \Sigma_{2}$-Replacement.) Hence, letting $S^{*}$ be the set of limit points of $S, S^{*}$ also has order type $\zeta+1$ (as $\zeta \in S^{*}$ ). And so for $\xi \in S^{*}$, $L_{\xi} \vDash \Sigma_{1}$-Separation.

Since we have a canonical $\Sigma_{2}^{L_{\zeta}}$ definable partial function $g_{\zeta} ; \omega \longrightarrow \zeta$ which is onto, for any $\alpha<\zeta$ if $n_{\alpha}$ is such that $g_{\zeta}\left(n_{\alpha}\right)=\alpha$, the statement $\Phi_{\alpha}$ : " $n_{\alpha} \in \operatorname{dom}(g)$ " is part of the $\Sigma_{2}$-theory of $L_{\zeta}$, which itself is true in some $L_{\rho(\alpha)}$ onwards. By Lemma 3.2(ii), for $\xi \in S^{*}$ the $\Sigma_{2}$-theory of $L_{\xi}$ is uniformly recursive in $F_{\xi}$, ( $L_{\xi}$ being a model of $\Sigma_{1}$-Separation). So let $G$ be (1-1) and recursive witnessing that $T_{\xi}^{2} \leq_{1} F_{\xi}$ for any such $\xi$.

We thus have that:

Claim $T_{\zeta}^{2}=\bigcup_{\xi \in S^{*} \cap \zeta} T_{\xi}^{2}=\bigcup_{\xi \in S^{*} \cap \zeta} G^{-1 “ F_{\xi}}$

Proof: The second equality expresses simply the remarks above about $G$ relating the relevant theories. The first equality is valid since $\Sigma_{2}$ sentences are absolute upwards from $L_{\xi}$ to $L_{\zeta}$ for any $\xi \in S$ : suppose $\varphi \equiv \exists u \forall \nu \psi(u, v)$ a $\Sigma_{2}$ sentence, and that $L_{\xi} \vDash \exists u \forall v \psi(u, v)$. Then let $u_{0} \in L_{\xi}$ be such that $L_{\xi}=\forall \nu \psi\left(u_{0}, v\right)$. Now we have a $\Pi_{1}$ formula about $u_{0}$ and such is upwards absolute as $L_{\xi} \prec_{\Sigma_{1}}$ $L_{\zeta}$, and so is true in $L_{\zeta}$. This ensures that $T_{\zeta}^{2} \supseteq \bigcup_{\xi \in S^{*} \cap \zeta} T_{\xi}^{2}$. Conversely if for some $\Sigma_{2} \varphi \equiv \exists u \forall v \psi(u, v), L_{\zeta} \vDash \varphi$ then again there will be some $\xi \in S^{*} \cap \zeta$ with $L_{\xi} \vDash \varphi$ : one simply has to find a sufficiently large $\xi \in S^{*}$ with $u_{0} \in L_{\xi}$ where $L_{\zeta}=\forall \nu \psi\left(u_{0}, v\right)$.

Q.E.D. Claim

With this, given the definition of $\Phi_{\alpha}$ above, we see that it is true in $L_{\xi(\alpha)}$ upwards where $\xi(\alpha)$ is the least element of $S^{*}$ greater than $\alpha$. Let then $B_{\alpha}$ be $G\left(\Phi_{\alpha}\right)$. Then any sentence $B_{\alpha}$ has stabilized by stage $\xi(\alpha)$ at the latest (and $\left.\left\|B_{\alpha}\right\|=1\right)$ ). 
Hence the order type of $<$ is no less than that of $\left\{\alpha \mid \alpha \in S^{*}\right\}$. But the latter we have remarked has order type $\zeta$. This concludes the Lemma.

Q.E.D. Lemma 1.6

The argument of the above proof shows that, in contrast to Theorem 1.2, we can regard $F_{\zeta}$ as a simple union, but only along a select subset of $\zeta$ :

Corollary 3.7 $F_{\zeta}=\bigcup_{\xi \in S^{*} \cap \zeta} F_{\xi}$.

Proof: We may imagine running the Fieldian construction inside $L_{\Sigma}$. Since the operations involved are highly absolute, we shall have $k \in F_{\alpha} \Leftrightarrow L_{\zeta} \vDash " k \in F_{\alpha}$ ". As " $k \in F_{\alpha}$ " is a $\Sigma_{2}$ sentence, the Claim of the last proof yields this result. Q.E.D.

\section{Proof of Lemma 1.7}

Let $Q\left(\nu_{0}, v_{1}\right)$ be a formula of $\mathscr{L}^{+}$. Define $n<_{Q} m$ if $\|Q(n, m)\|=1$. Suppose $\prec_{Q}$ is a prewellorder and for a contradiction that ot $\left(<_{Q}\right)>\Delta_{0}$. Let $m_{0} \in \mathbb{N}$ have rank $\Delta_{0}=\zeta$ in $\prec_{Q}$. Define $\prec_{\beta}$ to be the relation: $n<_{\beta} m$ if $|Q(n, m)|_{\beta}=1$. It is by assumption bivalent whether for any other $n \in \mathbb{N}$, that $Q\left(n, m_{0}\right)$ holds. Hence we have that for $\zeta<\beta$, for any $n \in \mathbb{N}, n \prec_{\beta} m_{0} \leftrightarrow n \prec_{Q} m_{0}$. Then for $\beta \in(\zeta, \Sigma)$ " $\zeta=\mathrm{rk}_{<_{\beta}}\left(m_{0}\right)$ " holds (where $\mathrm{rk}_{<_{\beta}}$ is the ranking function on (the wellfounded part of) $<_{\beta}$ ). Hence $\zeta$ is $\Pi_{1}$-definable in $L_{\Sigma}$ from $Q, m_{0}$ :

$z=\zeta \leftrightarrow \forall \tau>z$ ("if $\tau=\tau_{\iota}$ and $<_{\iota}, \mathrm{rk}_{<_{\iota}}$ are defined over $L_{\tau_{\iota}}$ then $z=\mathrm{rk}_{<_{\iota}}\left(m_{0}\right)$ "). But $L_{\zeta} \prec_{\Sigma_{2}} L_{\Sigma}$ so $\zeta$ is not $\Sigma_{2}$-definable from integers in $L_{\Sigma}$. This is a contradiction.

QED Lemma 1.7.

Lemma 3.8 If $C_{0} \in$ Field $(\preceq)$ then it is a bivalent matter for any sentence $B$, whether $B \preceq C_{0}$.

Proof: Suppose $C_{0} \in$ Field( $\subseteq$ ).

$B \preceq C_{0}$ implies \|“ $\neg \exists \sigma_{B} \exists \rho\left[\sigma_{B}>\rho=\rho\left(C_{0}\right) \wedge\left|P_{\leq}\left(B, C_{0}\right)\right|_{\sigma_{B}} \neq 1\right]$ ” $\|=1$ whilst

$B \npreceq C_{0}$ implies \|“ $\neg \exists \sigma_{B} \exists \rho\left[\sigma_{B}>\rho=\rho\left(C_{0}\right) \wedge\left|P_{\leq}\left(B, C_{0}\right)\right|_{\sigma_{B}} \neq 1\right] ” \|=0$.

Using our translations outlined above, the statement within quotes in the last two lines, has an arithmetical translate about the $\left\langle\mathbb{N}, F_{\beta}\right\rangle$. For example, " $\rho=$ $\rho\left(C_{0}\right)$ " can be written out using the 'stability' formula $X\left(\nu_{0}\right)$ and corresponding $\tilde{A}_{X}\left(v_{0}\right)$; this can be used again in conjunction with " $\left|P_{\leq}\left(B, C_{0}\right)\right|_{\xi} \neq 1$ " which itself can also be written out as a fact about the Gödel numbers of $P_{\leq}, B$, and $C_{0}$, coded into $F_{\beta}$, for any $\beta \geq \xi$.

Q.E.D. 


\subsection{Ineffable Liars}

Corresponding to his determinateness predicates Field defines generalised liar sentences $L_{\xi}$ as $\neg D^{\xi}\left(\operatorname{Tr}\left(L_{\xi}\right)\right)$ by the usual diagonalising processes. As he shows on the initial segment of this hierarchy that he defines in [5], this satisfies the following:

$$
\begin{aligned}
& \left\|D^{\sigma}\left(L_{\xi}\right)\right\|=0 \text { for } \sigma>\xi, \text { and } \\
& \left\|D^{\sigma}\left(L_{\xi}\right)\right\|=\frac{1}{2} \text { for } \sigma \leq \xi .
\end{aligned}
$$

We shall generalise this here as follows. Define as above for any sentence $C$ :

$$
D^{C}(A) \equiv \forall B\left[P_{\prec}(B, C) \rightarrow\left(\forall y\left(y=\left\ulcorner D^{B}(A)\right\urcorner \rightarrow \operatorname{Tr}(y)\right)\right)\right] .
$$

To summarise, the order type of $\leq$ is precisely $\zeta$, so that we have notations for ordinals $\xi<\zeta$ using sentences $C$ which stabilize in semantic value at the point $\rho(C)=\xi$. We then iterate $D$ 'along' the prewellordering $\leq$ to reach $D^{C}$. We may then define liar sentences $L_{C}$ as $\neg D^{C}\left(\operatorname{Tr}\left(L_{C}\right)\right)$. Again these are still sentences of the language $\mathscr{L}^{+}$and they obey the above equations:

$$
\begin{aligned}
& \left\|D^{C}\left(L_{B}\right)\right\|=0 \text { if }\left\|P_{<}(B, C)\right\|=1 \text { and } \\
& \left\|D^{C}\left(L_{B}\right)\right\|=\frac{1}{2} \text { if }\left\|P_{\leq}(C, B)\right\|=1 .
\end{aligned}
$$

as the formulae on the right reflect precisely the facts that $\rho(B)<\rho(C)$ and $\rho(C) \leq$ $\rho(B)$. Just as the $\mathscr{L}^{+}$sentence $D^{C}(A)$ makes sense, so again does the generalised liar diagonal sentence $L_{C}$ whether or not $C \in$ Field( $(\preceq)$. These $L_{C}$ for $C \notin \operatorname{Field}(\preceq)$, as promised in the introduction, furnish examples of diagonalised sentences, the ineffable liars, whose defectiveness is not encompassed by any $D^{B}$ for $B$ genuinely in Field( $(\preceq)$.

Proposition 3.9 There are sentences $C \in \mathscr{L}^{+}$so that for any determinateness predicate $D^{B}$ with $B \in$ Field $(\preceq)\left\|D^{B}\left(L_{C}\right)\right\|=\frac{1}{2}$. Thus the defectiveness of $L_{C}$ is not measured by any such determinateness predicate definable within the $\mathscr{L}^{+}$language.

Proof: We recall the fact that for the first two acceptable points in the models' construction $\zeta, \Sigma$ (in Field's notation more sensibly $\Delta_{0}, \Delta_{1}$ ) we have that $L_{\zeta}<_{2}$ $L_{\Sigma}$ (" $L_{\zeta}$ is a $\Sigma_{2}$-elementary substructure of $L_{\Sigma}$ " where $L_{\alpha}$ is the $\alpha$ 'th level of the Gödel constructible hierarchy.) Further, as $\mathbb{N} \in L_{\omega+1}$ and the successive levels of Field's construction are performed using very absolute processes, we 
may consider running the construction internally to the $L$-hierarchy. The ordinals $\zeta, \Sigma$ are highly closed, and in fact $\zeta$ is highly admissible. We set $\mathrm{ADM}^{+}=$ $\mathrm{ADM} \cap \mathrm{ADM}^{*}$ to be the class of admissible limits of admissible ordinals, We may define predicates in the language of set theory that give us the range of semantic values of sentences along Field's iteration. So that, if $\tau \in \mathrm{ADM}^{+}$then $\left(|A|_{\gamma}=\right.$ $i)_{L_{\tau}} \leftrightarrow|A|_{\gamma}=i$, in short that is, the construction is absolute to $L_{\tau}$. The discussion of evaluations on p. 254 of [5] indicates what happens for small ordinal iterations of $D$ : if $\alpha<\sigma$ then $D^{\alpha}\left(L_{\sigma}\right)$ cycles through the values $\frac{1}{2}$ followed by an $\alpha$-sequence of 0's, and then a tail of 1's making a $\sigma$-sequence altogether, before looping around again. $D^{\sigma}\left(L_{\sigma}\right)$ will cycle through $\frac{1}{2}$, and then a $\sigma$-sequence of 0 's before repeating; finally $D^{\sigma+1}\left(L_{\sigma}\right)$ will be an initial $\frac{1}{2}$ at stage 0 , but thereafter always 0 . Hence $\left\|D^{\sigma+1}\left(L_{\sigma}\right)\right\|=0$, and thus the 'defectiveness' of $L_{\sigma}$ is affirmed by this sentence. Essentially the same picture is intended for these extended operators, where now $\alpha, \sigma$ etc. are replaced by sentences $B, C, \cdots$ as notations.

(1) There are ordinals $\Sigma>\gamma>\xi>\zeta$ and a sentence $C$ with $\gamma \in \mathrm{ADM}^{+}$and $L_{\gamma}=“ \rho(C)=\xi$."

Proof: If not, then the following is true in $L_{\Sigma}$ :

$$
\begin{aligned}
& y=\zeta \leftrightarrow y \in \mathrm{ADM}^{+} \wedge L_{y} \vDash “ \forall \xi \exists C(\rho(C)=\xi) ” \wedge \\
& \wedge \forall y^{\prime} \in \operatorname{ADM}^{+}\left(y^{\prime}>y \longrightarrow L_{y^{\prime}} \models^{\prime} \forall C(\rho(C) \downarrow \longrightarrow \rho(C) \leq y)\right) . "
\end{aligned}
$$

Being in $\mathrm{ADM}^{+}$is a $\Delta_{1}$ notion, as are the satisfaction relations involving $L_{y}, L_{y^{\prime}}$. We note that $\zeta \in \mathrm{ADM}^{+}$. The second conjunct holds since $\mathrm{rk}(\preceq)=\zeta$, and all $B \in \operatorname{Field}(\preceq)$ have stabilized by stage $\zeta$. The last conjunct is our hypothesis. However this would imply that $\zeta$ is $\Pi_{1}$ definable (by the above definition) without using any other parameters in $L_{\Sigma}$ ). But it is not: only sets in $L_{\zeta}$ can be $\Sigma_{2}$ definable without parameters in $L_{\Sigma}$ (since $L_{\zeta}<_{\Sigma_{2}} L_{\Sigma}$ ). It particular $\zeta$ itself is not so definable.

Q.E.D.(1)

Let $C$ be as guaranteed in (1). Let $\bar{\zeta}<\zeta$ be arbitrary. Then we have (as a restatement, and weakening, of the above):

(2) $L_{\Sigma} \vDash=“ \exists \gamma \in \operatorname{ADM}^{+}\left(L_{\gamma} \vDash \rho(C)>\bar{\zeta}\right)$."

By $\Sigma_{1}$-elementarity then:

(3) $L_{\zeta} \vDash “ \exists \gamma \in \operatorname{ADM}^{+}\left(L_{\gamma} \vDash \rho(C)>\bar{\zeta}\right) "$. 
But $\bar{\zeta}$ was arbitrarily large below $\zeta$, thus, in fact:

(4) $L_{\zeta} \vDash " \forall \bar{\zeta} \exists \gamma>\bar{\zeta}\left(\gamma \in \mathrm{ADM}^{+} \wedge L_{\gamma} \vDash \rho(C)>\bar{\zeta}\right)$ ".

The claim is that, staying with this $C$, that it satisfies the proposition. Pick any $B \in \operatorname{Field}(\preceq)$. It suffices to show that

(5) $\forall \bar{\tau}<\zeta \exists \tau>\bar{\tau}\left(\tau<\zeta \wedge\left|D^{B}\left(L_{C}\right)\right|_{\tau} \neq 0\right)$.

Proof (5): Taking $\bar{\tau}$ any ordinal greater than $\rho(B)$, then by (3) (with $\bar{\tau}$ as $\bar{\zeta}$ there) there is $\gamma \in \mathrm{ADM}^{+}$with $L_{\gamma} \vDash \rho(C)>\rho(B)$. By choice, $\gamma$ is an admissible limit of admissibles, so $\gamma$ iterations of the Fieldian construction can be effected inside $L_{\gamma}$. But then inside $L_{\gamma}$ we see the usual picture of the cycling semantic values of $\frac{1}{2}, 0,0, \ldots$ (for $\rho(B)$ steps) and 1's for $\rho(C)-\rho(B)$ steps, then repeating this pattern. Consequently, with $\tau=\gamma$ we see $\left|D^{B}\left(L_{C}\right)\right|_{\tau} \neq 0$. $\quad$ Q.E.D.(5) \& Proposition.

In fact we can say a little more about such a $C$ : (4) is a $\Pi_{2}$ sentence about $C$, true in $L_{\zeta}$ and so goes up to be true in $L_{\Sigma}$. So for such a $C$, it attains arbitrarily large $\leq$-ranks, but locally in varying $L_{\gamma}$, and then only intermittently, as the construction proceeds. One may call such a $C$ sporadic. The non-stabilizing sentences in Field's model are of two kinds: those that exhibit a periodic behaviour with some fixed period $\xi<\zeta$, (and for every $\xi<\zeta$ there will be such) and the sporadics like $C$, which have no periodic behaviour at all below $\Sigma$ : if we want to assign a 'period' to $C$ it has to be $\Sigma$ itself (for which note that $\operatorname{ot}(\Sigma \backslash \zeta)=\Sigma$ ).

There is an entirely analogous result for the Herzberger sequence: in essence this is only a notational variant of the above. This is done in detail in [13]. Thus the defectiveness and determinateness hierarchy phenomena can be replicated in a Herzberger sequence. (This shows that they may be effectively decoupled from any notion of conditional operator such as Field's $\longrightarrow$.)

\section{Proof of the Limit Lemmata}

In this section we prove the $H$ - and $F$-Limit Lemmata. We have alluded to various set-theoretical facts about the $L$-hierarchy that are needed to prove these. We have to establish these here. For those familiar with the Gödel $L$-hierarchy, at least the statements of these facts should be understandable and indeed the proofs use only somewhat elementary concepts.

For those familiar with the Jensen $J$-hierarchy we make some comments 
now: Because the $H$-hierarchy is about iterated definability it is convenient to eschew the $J$-hierarchy and use the $L_{\alpha}$ since these are also created by iterated definability, and their ordinal height grows in step with the $H_{\alpha}$ (the ordinals heights of the $J_{\alpha}$ grow in multiples of $\omega$ : On $\cap J_{\alpha}=\omega . \alpha$ ). However the well known lack of closure of the $L_{\alpha}$ under even the most basic set theoretical constructs such as ordered pair, makes for difficulties. In particular we essentially justify in these lemmata the existence of uniform $\Sigma_{2}$-skolem functions for limit levels $L_{\lambda}$. Such skolem functions do not exist in general even for the $J_{\alpha}$-hierarchy, and for the $L_{\alpha}$ hierarchy are usually not defined. One has to justify the existence of such functions even using the $J_{\alpha}$ 's. The arguments here are in essence, modifications of those for the $J_{\alpha}$ 's run in [8].

Usually the existence of such functions is problematic for even moderate sized $\lambda$, and in general uniform versions do not exist. However, as mentioned in the first section, since we work below the ordinal $\beta_{0}$, it turns out that we are sufficiently low down in the $L$-hierarchy, so that all is well. This will cause some difficulties for us, but one thing works in our favour which is that we need only prove the existence of skolem functions, and our results, for limit $\lambda$ and the structures $\left\langle L_{\lambda}, \epsilon\right\rangle$.

\subsection{Proof of the $H$-Limit Lemma 2.5}

Throughout this proof $\lambda$ will denote a limit ordinal less than $\Sigma$. For such $\lambda$ we have a function $h_{\lambda}$ which is $\Sigma_{1}$-skolem function for $L_{\lambda}$. These are defined as follows.

Let $\left\langle\varphi_{n} \mid n<\omega\right\rangle$ be a recursive enumeration of all $\Sigma_{1}$ formulae in $\mathscr{L}_{\dot{E}}$ with say $\varphi_{n}=\varphi_{n}\left(\nu_{0}, v_{1}, \ldots, v_{m_{n}}\right)$ with free variables amongst those displayed. Let $\alpha \in$ On.

$$
\begin{aligned}
h_{\alpha}\left(n,\left\langle x_{1}, \ldots, x_{m_{n}}\right\rangle\right) & =y \Longleftrightarrow L_{\alpha}=\varphi_{n}\left[y, x_{1}, \ldots, x_{m_{n}}\right] \wedge \forall z<L_{\alpha} y \neg \varphi_{n}\left[z, x_{1}, \ldots, x_{m_{n}}\right] \\
& =\uparrow \text { (meaning undefined) otherwise. }
\end{aligned}
$$

We treat the right hand side as a definition of the left.

Moreover for any limit $\lambda$, the definition of $h_{\lambda}$, it turns out, is itself $\Sigma_{1}$ and one can establish that it has the same definition over any $L_{\lambda^{\prime}}$ for any limit $\lambda^{\prime}$. The existence of such uniform $\Sigma_{1}$-skolem functions for $L_{\lambda}, \lambda$ a limit, is justified in the same way as over every level of the $J$-hierarchy (as introduced in [14], and exposited in [3]; the arguments for the $J_{\alpha}$-hierarchy work here too). By considering only limit levels each $L_{\lambda}$ is closed under finite iterations of the pairing function as we have mentioned. Hence if $x_{1}, \ldots, x_{m_{n}} \in L_{\lambda}$ so is $\left\langle x_{1}, \ldots, x_{m_{n}}\right\rangle$ and the above then makes sense. The right hand side is defined using $<_{\alpha}$, a wellorder 
of $L_{\alpha}$ defined in a canonical fashion, but again for successor $\alpha$ this may only be defined over some later level, such as $L_{\alpha+5}$. For limit $\lambda$ however, all is well, and the wellorder $<_{\lambda}$ is then $\Delta_{1}$ over $L_{\lambda}$. We thus shall have:

$$
\begin{aligned}
& \forall \vec{x}=x_{1}, \ldots, x_{m_{n}}: \\
& \quad \exists x_{0} L_{\lambda} \vDash \varphi_{n}\left[x_{0}, x_{1}, \ldots, x_{m_{n}}\right] \longrightarrow L_{\lambda} \vDash \varphi_{n}\left[h_{\lambda}\left(n,\left\langle x_{1}, \ldots, x_{m_{n}}\right\rangle\right), x_{1}, \ldots, x_{m_{n}}\right] .
\end{aligned}
$$

Moreover the definition of $\Sigma_{1}$-satisfaction again can be shown to be a uniformly $\Sigma_{1}$-definable relation of $m$-tuples and (codes of) $\Sigma_{1}$-formulae over any limit $L_{\lambda}$. Thus for any $X \subseteq L_{\lambda}$ the range of $h_{\lambda}$ on $\omega \times[X]^{<\omega}$ is a $\Sigma_{1}$-elementary substructure of $\left\langle L_{\lambda}, \epsilon\right\rangle$, and in fact is the least $\Sigma_{1}$-skolem hull of $X$ in $\left\langle L_{\lambda}, \epsilon\right\rangle$.

For any ordinal $\alpha$ we may further define the set of ordinals $\beta<\alpha$ with $\left\langle L_{\beta}, \epsilon\right\rangle\left\langle\Sigma_{1}\right.$ $\left\langle L_{\alpha}, \epsilon\right\rangle$; this is the set of ordinals $\Sigma_{1}$-stable in $\alpha$, which we shall write as $S_{\alpha}^{1}$. This notation means that for any $\Sigma_{1}$-formula $\varphi_{n}$ and any $x_{0}, x_{1}, \ldots, x_{m_{n}} \in L_{\beta}$ if $\left\langle L_{\alpha}, \epsilon\right\rangle \vDash \varphi_{n}\left[x_{0}, x_{1}, \ldots, x_{m_{n}}\right]$ then $\left\langle L_{\beta}, \epsilon\right\rangle \vDash \varphi_{n}\left[x_{0}, x_{1}, \ldots, x_{m_{n}}\right]$. For this section we revert to the standard terminology that $L_{0}=\varnothing$. Then notice that $\beta \Sigma_{1}$-stable in $\alpha$ implies that $\beta=0$ or is a limit ordinal (consider the $\Sigma_{1}$ formula " $\exists y(\forall z \in y(z=$ $\gamma \vee z \in \gamma)$ )" which shows that $\beta$ cannot be $\gamma+1$ ).

“ $\alpha \in S_{\lambda}^{1}$ ” is a $\Pi_{1}$-predicate when defined over $L_{\lambda}$; again uniformly for any $\lambda$ (the uniformity uses the underlying uniformity of the $\Sigma_{1}$-skolem function). One should note that $\alpha \in S_{\lambda}^{1} \longrightarrow \alpha \in S_{\gamma}^{1}$ for any $\gamma \in(\alpha, \lambda]$ by the upwards persistence of $\Sigma_{1}$ properties from $\left\langle L_{\alpha}, \epsilon\right\rangle$ to $\left\langle L_{\lambda}, \epsilon\right\rangle$.

We restate what we are trying to prove here:

(H-Limit Lemma) For limit $\lambda \leq \Sigma$ the $\Sigma_{2}$-theory of $\left\langle L_{\lambda}, \epsilon\right\rangle, T_{\lambda}^{2}$, is re. in $\widehat{T}_{\lambda}$. Moreover an index for this re. reduction is the same for all such $\lambda$.

We now re-run the argument from Lemma 1 [8], but now for the $L$-hierarchy. Let $\varphi \equiv \exists x \psi(x)$ be $\Sigma_{2}$ where $\psi$ is taken as $\Pi_{1}$.

$$
\begin{aligned}
& \text { Claim }\left\langle L_{\lambda}, \epsilon\right\rangle \vDash \varphi \Longleftrightarrow \exists i \text { [for all sufficiently large } \alpha<\lambda \text { : } \\
& \qquad\left\langle L_{\alpha}, \epsilon\right\rangle \vDash “ \exists \beta \in S_{\alpha}^{1}\left(( \beta \neq 0 \wedge L _ { \beta } = \varphi ) \vee \left(h_{\alpha}(i,\langle\beta\rangle) \downarrow \wedge \psi\left(h_{\alpha}(i,\langle\beta\rangle)\right)\right.\right. \text { ”]. }
\end{aligned}
$$

Note that the right hand side here is of the form that for some $i$ the $\Sigma_{2}$-theory of $L_{\alpha}$ eventually from some point on contains the sentence within quotation marks; this latter sentence we shall call $\sigma_{\varphi}(i)$. As $\varphi$ is an arbitrary $\Sigma_{2}$ sentence, this yields the Lemma, since we may express this as $\varphi \in T_{\lambda}^{2} \leftrightarrow \exists i\left\ulcorner\sigma_{\varphi}(i)\right\urcorner \in \hat{T}_{\lambda}^{2}$.

Proof of Claim: $(\Longrightarrow)$ Suppose the left hand side holds. Suppose $S_{\lambda}^{1}$ were unbounded in $\lambda$; then for some $\beta \in S_{\lambda}^{1}$ we should have $\left\langle L_{\beta}, \epsilon\right\rangle \vDash \varphi$ and thus $\forall \alpha \in$ $[\beta, \lambda]$ we should have $\left\langle L_{\alpha}, \epsilon\right\rangle \vDash \varphi$ by the above mentioned upwards persistence 
property. Hence the first disjunct of the right hand side holds. Otherwise let $\beta=$ $\max S_{\lambda}^{1}$ (which may be 0 ). We may consider, $H$, the $\Sigma_{1}$-skolem hull of $\{\beta\}$ in $\left\langle L_{\lambda}, \epsilon\right.$ \rangle . In this region of the $L$ hierarchy, for every level: $L_{\gamma}=$ "every set is countable", and consequently there is in $L_{\beta+1}$ a function $f: \omega \longrightarrow \beta$ which is onto. Moreover the $<_{L}$-least such $f$ is $\Delta_{1}$-definable from $\beta$. Consequently $\beta+1 \subseteq H$ (and so $\left.L_{\beta+1} \subseteq H\right)$. The same argument shows that $\gamma \in H \longrightarrow \gamma \subseteq H\left(\longrightarrow L_{\gamma} \subseteq H\right)$. Thus $H$ is transitive, and hence is $L_{\gamma}$ for some $\gamma \leq \lambda$. But notice that were $\gamma<\lambda$ then $\gamma \in S_{\lambda}^{1}$. But $\gamma>\beta$ so this is a contradiction. Thus $H=L_{\lambda}$. Hence every $x \in L_{\lambda}$ is of the form $h(n, \beta)$. But the equation $x=h(n,\langle\beta\rangle)$ being $\Sigma_{1}$ will hold for all sufficiently large $\alpha<\lambda$. If $i$ has been chosen so that the $\Pi_{1} \psi\left(h_{\alpha}(i,\langle\beta\rangle)\right)$ holds in $\lambda$ it will also again hold for all sufficiently large $\alpha<\lambda$, as the $\Pi_{1}$ statement persists downwards. We are thus done.

$(\Longleftarrow)$ Suppose the left hand side fails, but the right hand side holds. Then note that $S_{\lambda}^{1}$ is bounded in $\lambda$ : for otherwise we could apply the right hand side to an $\alpha$ in $S_{\lambda}^{1}$. However then if the first disjunct held for some $\beta \in S_{\alpha}^{1} \subseteq S_{\lambda}^{1}$ we should have $L_{\lambda} \vDash \varphi$, contradicting our assumption. If the second disjunct held then we have the same conclusion since $\alpha$ was chosen in $S_{\lambda}^{1}$. Hence we may set $\beta=\max S_{\lambda}^{1}$. This definition of $\beta$ ensures that there are arbitrarily large $\alpha<\lambda$ with $S_{\alpha}^{1} \subseteq \beta+1 \cap S_{\lambda}^{1}$. However this latter inclusion shows that again the first disjunct cannot be true for all sufficiently large $\alpha$, else $\left\langle L_{\lambda}, \epsilon\right\rangle \vDash \varphi$. So the second disjunct must hold instead. Choose $i$ witnessing this, and then for any $\alpha$ large enough take $\beta_{\alpha}$ so that $h_{\alpha}\left(i,\left\langle\beta_{\alpha}\right\rangle\right) \downarrow \wedge \psi\left(h_{\alpha}\left(i,\left\langle\beta_{\alpha}\right\rangle\right)\right.$ for some $\beta_{\alpha} \in S_{\alpha}^{1}$. If $\beta_{\alpha}$ were less than $\beta$ for such an $\alpha$ we'd have $h_{\alpha}\left(i,\left\langle\beta_{\alpha}\right\rangle\right) \downarrow \longrightarrow h_{\beta}\left(i,\left\langle\beta_{\alpha}\right\rangle\right)=$ $h_{\alpha}\left(i,\left\langle\beta_{\alpha}\right\rangle\right) \wedge L_{\beta} \vDash " h_{\beta}\left(i,\left\langle\beta_{\alpha}\right\rangle\right) \downarrow$ ", and moreover $\psi\left(h_{\beta}\left(i,\left\langle\beta_{\alpha}\right\rangle\right)\right.$ would be downwards absolute from $L_{\alpha}$ to $L_{\beta}$ also. Hence $\left\langle L_{\beta}, \epsilon\right\rangle \vDash \varphi$ and as $\beta \in S_{\lambda}^{1}$, we'd have $\left\langle L_{\lambda}, \epsilon\right\rangle \vDash \varphi$ - a contradiction. Hence $\beta_{\alpha}$ is always equal to $\beta$ : but as this is the case for all sufficiently large $\alpha<\lambda$ we should also have $L_{\alpha} \models^{\text {" }} \psi(x)$ " for such $\alpha$ where $x=h_{\alpha}(i,\langle\beta\rangle)$. But this again means $\left\langle L_{\lambda}, \epsilon\right\rangle \vDash \varphi$ - our final contradiction.

Q.E.D. Claim and Lemma 2.5.

\subsection{Proof of the existence of uniformly definable wellorderings}

Lemma 4.1 For any limit $\lambda<\Sigma$ there is a partial function $g: \omega \rightarrow L_{\lambda}$ that is onto which is itself $\Sigma_{2}$ definable over $L_{\lambda}$ (without parameters), and in a way that is independent of $\lambda$.

Proof: We assume a recursive enumeration $\left\langle\psi_{n}\left(v_{0}\right) \mid n<\omega\right\rangle$ of all $\Pi_{1}$ formulae of the one free variable $v_{0}$. Define 
$f^{\prime}(n)=\langle m, \beta\rangle$

iff the following hold in $L_{\lambda}$ :

(i) $\beta \in S_{\lambda}^{1}$;

(ii) $\exists x\left(x=h_{\lambda}(m,\langle\beta\rangle)\right)$ (thus $h_{\lambda}(m,\langle\beta\rangle)$ is defined);

(iii) $\psi_{n}[x]$;

(iv) $\forall \beta^{\prime}<\beta \forall m^{\prime}<\omega \forall x^{\prime}\left(x^{\prime}=h_{\lambda}\left(m^{\prime},\left\langle\beta^{\prime}\right\rangle\right) \longrightarrow \neg \psi_{n}\left[x^{\prime}\right]\right)$;

(v) $\forall m^{\prime}<m \forall x^{\prime}\left(x^{\prime} \neq h_{\lambda}\left(m^{\prime},\langle\beta\rangle\right)\right) \vee \exists x^{\prime}\left(x^{\prime}=h_{\lambda}\left(m^{\prime},\langle\beta\rangle\right) \wedge \neg \psi_{n}\left[x^{\prime}\right]\right)$.

All of the above statements are Boolean combinations of $\Sigma_{1}$ and $\Pi_{1}$ statements about their various parameters: (i) and (iii) are $\Pi_{1}$ about $\beta$ and $x$ respectively; (ii) is $\Sigma_{1}$. (iv) is vacuous if $\beta=0$ but otherwise it holds in $L_{\lambda}$ if and only if " $\forall m^{\prime}<\omega \forall x^{\prime}\left(x^{\prime}=h_{\beta}\left(m^{\prime},\left\langle\beta^{\prime}\right\rangle\right) \longrightarrow \neg \psi_{n}\left[x^{\prime}\right]\right)$ " holds in $L_{\beta}$. Hence over $L_{\lambda}$, it is a $\Sigma_{1}$ statement about $\beta$. (v) Is a finite quantifier in front of a statement saying that either $h_{\lambda}\left(m^{\prime},\langle\beta\rangle\right)$ is undefined, or else it is defined but $\psi$ is false of it. It is thus equivalent to a finite conjunction of disjunctions of $\Pi_{1}$ and $\Sigma_{1}$ statements. $f^{\prime}: \omega \longrightarrow L_{\lambda}$ and is $\Sigma_{2}$ definable over $L_{\lambda}$ without reference to any parameters.

Now set $f(n)=h_{\lambda}(m,\langle\beta\rangle)$ where $f^{\prime}(n)=\langle m, \beta\rangle$. Let $H$ be the $\Sigma_{1}$-skolem hull of $\operatorname{ran}(f)$. Then $H$ can be realised as the set of all objects of the form $h_{\lambda}\left(i,\left\langle f\left(n_{1}\right), \ldots, f\left(n_{k}\right)\right\rangle\right.$. Using a recursive coding of tuples from $\mathbb{N}$ with $\mathbb{N}$, if $n$ codes $\left\langle i, n_{1}, \ldots, n_{k}\right\rangle$, we may set $g(n)=h_{\lambda}\left(i,\left\langle f\left(n_{1}\right), \ldots, f\left(n_{k}\right)\right\rangle\right) ; g$ is thus a partial map from $\omega$ onto $H$. Again $g$ is $\Sigma_{2}$ definable (from the underlying $f^{\prime}$ ) over $L_{\lambda}$ without parameters. Being a $\Sigma_{1}$ skolem hull, $H$ is in fact a $\Sigma_{1}$-elementary substructure of $L_{\lambda}$. We claim it is more:

Claim $\mathrm{H}$ is a $\Sigma_{2}$-elementary substructure of $L_{\lambda}$.

Proof of Claim: Let $g$ from above have the $\Sigma_{2}$ defining formula $g(n)=x \leftrightarrow\left\langle L_{\lambda}, \epsilon\right\rangle \vDash \exists u \Phi(u, n, x)$ where $\Phi$ is $\Pi_{1}$.

Let, for simplicity, $\left.\left\langle L_{\lambda}, \epsilon\right\rangle \vDash \exists v \psi(\nu, g(n))\right)$ be a $\Sigma_{2}$ statement about the single parameter $g(n)$ from $H$ (the argument with further parameters in $\psi$ is only notationally longer). We need to show that $\langle H, \epsilon\rangle \models \exists v \psi(\nu, g(n))$. Pick $z$ so that $\left.\left\langle L_{\lambda}, \epsilon\right\rangle \vDash \psi(z, g(n))\right)$ and $u$ so that $\left\langle L_{\lambda}, \epsilon\right\rangle \vDash \Phi(u, n, x)$.

Thus $\left\langle L_{\lambda}, \epsilon\right\rangle \vDash \Phi(u, n, x) \wedge \psi(z, x)$.

The latter can be rewritten as a $\Pi_{1}$ formula about $\langle u, z, x\rangle$; it is thus of the form $\psi_{k}\left(v_{0} /\langle u, z, x\rangle\right)$ where $\psi_{k}$ is from our original list. As $L_{\lambda}=h_{\lambda}$ “ $\omega \times S_{\lambda}^{1}$, so there is $f^{\prime}(k)=(m, \beta)$ satisfying (i)-(v) above, with $h_{\lambda}(m,\langle\beta\rangle)=\left\langle u^{\prime}, z^{\prime}, x^{\prime}\right\rangle$ so that $\psi_{k}\left(\nu_{0} /\left\langle u^{\prime}, z^{\prime}, x^{\prime}\right\rangle\right)$, but now with $\left\langle u^{\prime}, z^{\prime}, x^{\prime}\right\rangle \in \operatorname{ran}(f) \subseteq H$. But this means

$\left\langle H_{\lambda}, \epsilon\right\rangle=\Phi\left(u^{\prime}, n, x^{\prime}\right) \wedge \psi\left(z^{\prime}, x^{\prime}\right)$

and thus, as $g(n)=x=x^{\prime}$

$\left.\left\langle H_{\lambda}, \epsilon\right\rangle \mid=\exists v \psi(\nu, g(n))\right)$,

and so we are done.

Q.E.D. Claim 
However by our definition of $\Sigma$ the only $\Sigma_{2}$-elementary substructure of $L_{\lambda}$ is $L_{\lambda}$ itself. In other words $H=L_{\lambda}$ and $g$ is our required partial onto map needed to fulfill the Lemma.

Q.E.D. Lemma 4.1

Proof of Corollary 2.10. This is the main part of the proof of the last lemma: $g$ is a partial map from $\omega$ onto $\lambda$ which has a $\Sigma_{2}^{L_{\lambda}}$ definition. In that definition, no individual property of $\lambda$ was used; hence it is independent of $\lambda$. Thus such a wellorder $w_{\lambda}$ for the Corollary is recursive in $T_{\lambda}^{2}$ which is in turn r.e. in $\widehat{T}_{\lambda}^{2}$, by Lemma 2.5. Finally the latter is r.e. in $H_{\lambda}$ and hence is $\Sigma_{1}^{H_{\lambda}}$. From this a $G$ as in the Corollary is easily defined.

Q.E.D. Cor. 2.10

This process holds together for as long as new $\Sigma_{2}$-theories of $L_{\alpha}$ 's are produced. However when we reach $\Sigma$, then $\Sigma_{2}$ - Th $\left(\left\langle L_{\Sigma}, \epsilon\right\rangle\right.$ equals $\Sigma_{2}$ - Th $\left(\left\langle L_{\zeta}, \epsilon\right\rangle\right.$ (because $\left.L_{\zeta}<_{\Sigma_{2}} L_{\Sigma}\right)$ and it cannot construct a code $r_{\Sigma}$ for $L_{\Sigma}$ from it, and the process breaks down. But that of course is the underlying reason that the Herzberger revision process cycles back at $H_{\Sigma}$ to $H_{\zeta}$.

Proof of Lemma 3.3 (F-Limit Lemma I) For a limit $\lambda$ the $\Sigma_{2}$ theory of $\left\langle L_{\tau_{\lambda}}, \epsilon\right\rangle, T_{\tau_{\lambda}}^{2}$, is r.e. in $F_{\lambda}$. Moreover an index for this re. reduction is uniform in $\lambda$.

This follows from the fact that $F_{\lambda}$ is the $\liminf _{\alpha \rightarrow \lambda} F_{\alpha}$. Consequently just as $T_{\lambda}^{2}$ could be found by the argument of the proof of Lemma 2.5's Claim from $H_{\lambda}$, in an r.e. fashion, $T_{\tau_{\lambda}}^{2}$ can be similarly obtained from $F_{\lambda}$. Again no particular properties of $\lambda$ are used.

Q.E.D. Lemma 3.3

Hence $T_{\tau_{\lambda}}^{2}$ is $\Sigma_{1}\left(\left\langle\mathbb{N}, F_{\lambda}\right\rangle\right)$.

Proof of Corollary 3.4 (F-Wellordering Lemma) (cf. [8])

Again this corollary follows in the same fashion as the existence of the function $G$ in Corollary 2.10 .

Q.E.D.

\section{Proof of Lemma 2.7}

Lemma 2.7 There is a recursive (1-1) function $G_{2}$ so that for $\operatorname{Succ}(\alpha), T_{\alpha}$ is (1-1) in $\left(T_{\alpha-1}\right)^{(\omega)}$ with: $T_{\alpha}=G_{2}^{-1}$ “ $\left(T_{\alpha-1}\right)^{(\omega)}$.

Proof:

(1) A code $r_{\alpha-1}$ for $\left\langle L_{\alpha-1}, \epsilon\right\rangle$ is uniformly definable from $T_{\alpha-1}$. In fact for some fixed $N<\omega$, it is uniformly recursive in $T_{\alpha-1}^{N+1}$. 
Proof (1)

Proof: For $\operatorname{Lim}(\beta)$ we saw that by Lemma 4.1 there is a uniform $\Sigma_{2}^{L_{\beta}}$ definable map $f_{\beta}: \omega \rightarrow L_{\beta}$ for $\beta<\Sigma$ which is essentially, modulo some pairing, the uniform $\Sigma_{2}^{L_{\beta}}$-skolem fumction which we have at these levels. We then set:

$\left\langle n_{0}, n_{1}\right\rangle \in r_{\beta} \leftrightarrow$

$\leftrightarrow\left\langle L_{\beta}, \epsilon\right\rangle \vDash f\left(n_{0}\right) \in f\left(n_{1}\right) \wedge \forall n<n_{0} \forall m<n_{1}\left[n \in \operatorname{dom}(f) \longrightarrow f(n) \neq f\left(n_{0}\right) \wedge\right.$ $\left.m \in \operatorname{dom}(f) \longrightarrow f(m) \neq f\left(n_{1}\right)\right]$.

We thus are singling out a least element to name $f\left(n_{0}\right)$ etc. This is $\Sigma_{2} \wedge \Pi_{2}$ definable over $L_{\beta}$. Hence is recursive in $T_{\beta}^{3}$ (uniformly in such limit $\beta$ ) we have an arithmetic copy or code of $L_{\beta}$. We can run the above argument if we have a function $f_{\beta+k}$ uniformly definable in $\left(\beta\right.$ and $k$ ) over $L_{\beta+k}$ such that $f_{\beta+k}: \omega \rightarrow$ $L_{\beta+k}$.

As is well known, for successor ordinals of the form $\beta+k$ for $0<k<\omega, L_{\beta+k}$ is not a terribly suitable model for many of these arguments. For example, it is not closed under Kuratowski pairs. In Devlin [3] often the assumption of $\beta$ being a limit is made, in order to simply define many of the known concepts of $L$, such as the existence of a definable wellorder $<_{\beta}$, definable over $L_{\beta}$, and the existence of $\Sigma_{1}$-definable $\Sigma_{1}$-skolem functions. However Boolos in [1] addresses the problems of defining the necessary concepts uniformly for all $\beta$. He firstly uses Quinean pairing rather that Kuratowski pairs, to define a notion of finite sequence that does not raise constructibility rank, so that for any $x_{1}, \ldots, x_{n} \in L_{\beta+k}$, $\left\langle\left\langle x_{1}, \ldots, x_{n}\right\rangle\right\rangle \in L_{\beta+k}$. This pairing $\langle\langle\cdots\rangle\rangle$ is moreover absolute when defined over any $L_{\alpha}$. We use here his (I)-(III)

(Ia) The notion of $b$ being first order definable over $c$ can be formalised as " $b$ fodo $c$ ", and is absolute when defined over any $L_{\alpha}$.

(Ib) There is a sentence Close which is true in any transitive set, and implies when true in a set $t$ that it is sufficiently closed, so that if $c \in t$ and $b$ fodo $c$, then $b \in t$.

(II) There is a sentence $\sigma$ so that for any wellfounded model $\langle R, E\rangle$, it is a model of $\sigma$ iff $\exists \alpha \geq \omega\left(\langle R, E\rangle \cong\left\langle L_{\alpha}, \epsilon\right\rangle\right)$.

(III) There is a binary predicate $C\left(\nu_{0}, v_{1}\right)$ that defines over any $L_{\alpha}$ a wellordering $<_{\alpha}$ of $L_{\alpha}$ with the usual property that $\alpha<\beta \longrightarrow<_{\alpha}$ is end-extended by $<_{\beta}$.

Now granted the above, suppose the definitions to be uniformly $\Sigma_{N-1}^{L_{\alpha}}$ for some sufficiently large $N$. We may thus for any $L_{\beta+k}$ define a $\Sigma_{N}$-skolem function $h_{\beta+k}^{\prime}$ with the property that $h_{\beta+k}^{\prime} " \omega \times \omega^{<\omega}=L_{\beta+k}$. (Here $\omega^{<\omega}$ denotes the finite sequences formed using $\langle\langle\cdots\rangle\rangle$.)

We do this in the most straightforward manner: define for limit $\beta \geq \omega, h_{\beta+k}^{\prime}(i, \vec{q}) \simeq<\beta+k^{-}$ least $x$ so that $\varphi_{i}(x, \vec{q})$, where $\varphi_{i}$ enumerates the $\Sigma_{N}$ formulae. By doing this we 
ensure that the skolem hull $X=h_{\beta+k}^{\prime} " \omega \times \omega^{<\omega}$ is a model of Close and $\sigma$. The transitive collapse of $X$ is then by (II), some $L_{\gamma}$ for a $\gamma \leq \beta+k$. We claim that $X=L_{\beta+k}$. Note that $\beta \in X$ as the largest limit ordinal is $\Pi_{1}^{L_{\beta+k}}$ definable. As $\beta$ is definably collapsed to $\omega$ over $L_{\beta+1}$ by a $\Sigma_{2}^{L_{\beta}}$-definable function, $g$ say, we have that $g$ is in $X$ and hence $\beta+k \subseteq X$. This suffices then.

By composing $g$ and $h^{\prime}$ with some (ordinary number) pairing we see then that there is a function $f_{\beta+k}: \omega \rightarrow L_{\beta+k}$. However $f_{\beta+k}$ and $h_{\beta+k}^{\prime}$ need not be $\Sigma_{2}$-definable over $L_{\beta+k}$, but they will be $\Sigma_{N+1}$ over $L_{\beta+k}$ uniformly in $\beta<\Sigma$ and $k<\omega$.

(2) Uniformly in $\alpha$, we may find a code $r$ for $\left\langle L_{\alpha}, \epsilon\right\rangle$ with $r \leq_{1}\left(r_{\alpha-1} \oplus T_{\alpha-1}\right)^{(N+2)}$.

Proof (2): This mimics the usual construction of $L_{\alpha}$ as $L_{\alpha-1}$ together with the sets first order definable over $\left\langle L_{\alpha-1}, \epsilon\right\rangle$. Note that since everything in $L_{\alpha-1}$ is of the form $f(n)$ for some $n$, every element of $L_{\alpha}$ is definable by a parameterfree formula of a single variable. We assume therefore a recursive assignment of gödel numbers with $\left\ulcorner\varphi\left(\nu_{0}\right)\right\urcorner$ coding $\varphi\left(\nu_{0}\right)$, the latter any formula of LST with just the single free variable $v_{0}$. We set $T=T_{\alpha-1}, s=r_{\alpha-1}$. Define:

$$
\begin{gathered}
\left\langle n_{0}, 0\right\rangle E\left\langle n_{1}, i\right\rangle=\mathrm{df}\left(i=0 \wedge\left\langle n_{0}, n_{1}\right\rangle \in s\right) \vee\left(i=1 \wedge n_{1}=\left\ulcorner\varphi\left(\nu_{0}\right)\right\urcorner \wedge\left\ulcorner\varphi\left(\bar{n}_{0} / \nu_{0}\right)\right\urcorner \in T .\right. \\
\left\langle n_{0}, i\right\rangle \approx\left\langle n_{1}, j\right\rangle=\mathrm{df}\left(i=j=0 \wedge n_{0}=n_{1} \in \operatorname{Field}(s)\right) \vee \\
\vee\left[n_{0} \in \operatorname{Field}(s) \wedge j=1 \wedge n_{1}=\left\ulcorner\varphi\left(\nu_{0}\right)\right\urcorner\right. \\
\left.\left.\wedge \forall m \in \operatorname{Field}(s)\left(\left\langle m, n_{0}\right\rangle \in s \leftrightarrow\left\ulcorner\varphi\left(\bar{m} / \nu_{0}\right)\right)\right\urcorner \in T\right)\right] \vee \\
\vee\left[i=j=1 \wedge n_{0}=\left\ulcorner\varphi_{0}\left(\nu_{0}\right)\right\urcorner \wedge n_{1}=\left\ulcorner\varphi_{1}\left(\nu_{0}\right)\right\urcorner \wedge\right. \\
\left.\wedge \forall m \in \operatorname{Field}(s)\left(\left\ulcorner\varphi_{0}\left(\bar{m} / \nu_{0}\right) \leftrightarrow \varphi_{1}\left(\bar{m} / \nu_{0}\right)\right\urcorner \in T\right)\right] .
\end{gathered}
$$

Then $E$ and $\approx$ are (1-1) in $(s \oplus T)$. Let $U=\{[\omega \times 2] \approx\}$, the set of $\approx$ equivalence classes, then

$$
\mathfrak{A}=\langle U, E\rangle \cong\left\langle L_{\alpha}, \epsilon\right\rangle .
$$

In particular if $\Phi_{f}$ defines the uniform $\Sigma_{N}^{L_{\alpha}}$ map $f_{\alpha}$ of $\omega$ onto the whole structure $L_{\alpha}$, we can replace $E$ by a code $r$ :

$$
\begin{aligned}
& \left\langle n_{0}, n_{1}\right\rangle \in r \leftrightarrow \\
& \langle\omega \times 2, E, \approx\rangle \models “\left\langle n_{0}, 0\right\rangle,\left\langle n_{1}, 0\right\rangle \text { are finite integers } \wedge f\left(\left\langle n_{0}, 0\right\rangle\right) \in f\left(\left\langle n_{1}, 0\right\rangle\right) \wedge \\
& \wedge \forall\langle n, 0\rangle<\left\langle n_{0}, 0\right\rangle \forall\langle m, 0\rangle<\left\langle n_{1}, 0\right\rangle\left[\langle n, 0\rangle \in \operatorname{dom}(f) \longrightarrow f(\langle n, 0\rangle) \neq f\left(\left\langle n_{0}, 0\right\rangle\right) \wedge\right. \\
& \left.\left.\langle m, 0\rangle \in \operatorname{dom}(f) \longrightarrow f(\langle m, 0\rangle) \neq f\left(\left\langle n_{1}, 0\right\rangle\right)\right)\right] . "
\end{aligned}
$$

Again we are using the same trick of taking 'least representatives'. This is $\Sigma_{N+1} \wedge \Pi_{N+1}$ in $E$ and $\approx$ and so the graph of $r$ is (1-1) reducible to $(s \oplus T)^{(N+2)}$. 
Q.E.D.(2)

Hence

(3) $T_{\alpha}^{k} \leq_{1}\left(r_{\alpha-1} \oplus T_{\alpha-1}\right)^{(N+2+k)}$.

By 1) we may absorb the $r_{\alpha-1}$ here. Then we have $T_{\alpha} \leq_{1}\left(T_{\alpha-1}\right)^{(\omega)}$. Q.E.D.

\section{Conclusions}

Is there a simpler way of proving the non-decreasing nature of the $H$-sets? (Probably if there was, this would work for the $F$-sets too.) In one sense the above argument is indirect: it does not principally use the definition of the $\mathrm{H}$ sets directly; but rather uses the $L_{\gamma}$-hierarchy of iterated definability. Possibly there is a direct argument. It might at first sight seem odd that it is difficult to show that the $H$-sets are non-decreasing with index, but that most simple way of ensuring this conclusion - by arguing that the stock of $\Sigma_{1}$-sentences in the $H_{\delta}$ must increase with index as new $\Sigma_{1}$ facts become true - cannot be deployed. This is because there are large stretches of ordinals $[\beta, \gamma] \subset \zeta$ where no new $\Sigma_{1}$ set-theoretical sentences become true in the $L_{\delta}$ for $\delta$ in the interval [ $\left.\beta, \gamma\right]$; this must happen by the nature of the ordinals $(\zeta, \Sigma)$. Since we may run a mirror of the revision process inside the $L$-hierarchy, and the membership in such internal $H$-sets and those constructed externally, is absolute, there will a fortiori, during those stretches [ $\beta, \gamma]$, be no new persisting $\Sigma_{1}$ truths entering the $H$-sets. So, that relatively simple argument is ruled out: we must step up to $\Sigma_{2}$ 'facts', and using the definable $\Sigma_{2}$ wellorderings seems then as good a way as any.

In the above we have concentrated on the ground model for $\mathscr{L}$ as $\mathscr{M}=\mathbb{N}$, the standard model of arithmetic. This is only for perspicuousness: almost any other model would be substitutable here: if the model contains a copy of the natural numbers, this is particularly easy. For models $\mathscr{M}=V_{\alpha}$ say, the set of all sets of rank less than a fixed $\alpha$ ( $\alpha$ not necessarily an cardinal) one may effect the above in at least two ways: either by assuming that the ground language $\mathscr{L}_{\mathscr{M}}$ contains a constant $c_{x}$ for every $x \in V_{\alpha}$, and then constructing an $H$ - or $F$ sequence over $\mathscr{M}$. This would have length the corresponding ordinal $\zeta(\mathscr{M})$ and would be least such that there is $\Sigma(\mathscr{M})$ with $L_{\zeta(\mathscr{M})}(\mathscr{M})<\Sigma_{2} L_{\Sigma(\mathscr{M})}(\mathscr{M})$. One may then use the arguments above to talk about determinateness paths of length up to this new $\zeta(\mathscr{M})$; ineffable liars can be constructed in such contexts.

Another approach is to add to the Tr predicate a satisfaction predicate (as for example Field indicates in his book for the $F$-model he builds, using "True- 
of"). This would again give rise to the same ordinals. For $\mathscr{M}=V_{\alpha}$ then these approaches yield uncountable ordinals $\zeta(\mathscr{M})>\alpha$. However for $\mathscr{M}$ not of this form, as long as we require that objects in $\mathscr{M}$ have names in the language $\mathscr{L}_{\mathscr{M}}$ and we may form diagonalising functions etc. then the above is all possible. The ideas above will suffice in these other contexts, by building the appropriate constructible hierarchies over the chosen $\mathscr{M}$. The notions of recursive and r.e. have to be abandoned for other appropriate forms of uniform definability.

\section{References}

[1] G. Boolos. On the semantics of the constructible levels. Zeitschrift für Mathematische Logik, 16:139-148, 1970.

[2] J.P. Burgess. The truth is never simple. Journal of Symbolic Logic, 51(3):663681, 1986.

[3] K. Devlin. Constructibility. Perspectives in Mathematical Logic. Springer Verlag, Berlin, Heidelberg, 1984.

[4] H. Field. A revenge-immune solution to the semantic paradoxes. Journal of Philosophical Logic, 32(3):139-177, April 2003.

[5] H. Field. Saving Truth from Paradox. Oxford University Press, 2008.

[6] H. Field. Solving the paradoxes, escaping revenge. In J.C. Beall, editor, The Revenge of the Liar. O.U.P., Oxford, 2008.

[7] H. Field. Responses at APA session Dec. 2009. Review of Symbolic Logic, 4(3), September 2011.

[8] S-D. Friedman and P.D. Welch. Two observations concerning infinite time Turing machines. In I. Dimitriou, editor, BIWOC 2007 Report, pages 44-47, Bonn, January 2007. Hausdorff Centre for Mathematics. Also at http://www.logic.univie.ac.at/sdf/papers/joint.philip.ps.

[9] A. Gupta and N. Belnap. The revision theory of truth. M.I.T. Press, Cambridge, 1993.

[10] J.D. Hamkins and A. Lewis. Infinite time Turing machines. Journal of Symbolic Logic, 65(2):567-604, 2000.

[11] H.G. Herzberger. Naive semantics and the Liar paradox. Journal of Philosophy, 79:479-497, 1982. 
[12] H.G. Herzberger. Notes on naive semantics. Journal of Philosophical Logic, 11:61-102, 1982.

[13] L. Horsten, G. Leigh, H. Leitgeb, and P.D. Welch. Revision Revisited. Review of Symbolic Logic, 2012.

[14] R. B. Jensen. The fine structure of the constructible hierarchy. Annals of Mathematical Logic, 4:229-308, 1972.

[15] S. Kripke. Outline of a theory of truth. Journal of Philosophy, 72:690-716, 1975.

[16] D. A. Martin. Revision and its rivals. Philosophical Issues, 8:407-418, 1997.

[17] D. A. Martin. Field's saving truth from paradox: some things it doesn't do. Review of Symbolic Logic, 4(3), Sep 2011.

[18] P-G. Odifreddi. Classical Recursion Theory: the theory of functions and sets of natural numbers. Studies in Logic. North-Holland, Amsterdam, 1989.

[19] R.I.Soare. Recursively enumerable sets and degrees. Perspectives in Mathematical Logic. Springer, 1987.

[20] H. Rogers. Recursive Function Theory. Higher Mathematics. McGraw, 1967.

[21] G.E. Sacks. Higher Recursion Theory. Perspectives in Mathematical Logic. Springer Verlag, 1990.

[22] P.D. Welch. Ultimate truth vis à vis stable truth. Review of Symbolic Logic, 1(1):126-142, June 2008.

[23] P.D. Welch. Characteristics of discrete transfinite Turing machine models: halting times, stabilization times, and normal form theorems. Theoretical Computer Science, 410:426-442, January 2009.

[24] P.D. Welch. Games for Truth. Bulletin of Symbolic Logic, 15(4):410-427, December 2009.

[25] P.D. Welch. Truth, logical validity, and determinateness: a commentary on Field's saving truth from paradox. Review of Symbolic Logic, 4(3), Sep 2011. 